LIAMES, Campinas, SP, v. 20, 1-45, e020014, 2020

\title{
La predicación nominal posesiva en la expresión de las relaciones interpersonales en las lenguas de la familia mataguayo (Gran Chaco), con énfasis especial en el nivaĉle y el maká
}

\author{
Alain Fabre \\ Investigador independiente, Finlandia \\ https://orcid.org/0000-0003-2738-0691
}

\begin{abstract}
The aim of this article is to describe how kinship and other interpersonal relations can be used in possessive nominal predication of two Mataguayo languages of the Chaco, Nivaĉle and Maká. It will focus on the subtype in which an utterance such as I am your son can be expressed with only one word, which stands out crosslinguistically as a quite uncommon construction. Additionally, some observations concerning similar phenomena in the other Mataguayo languages, Wichí/'Weenhayek and Chorote, are presented.

KEYwords: Chaco languages; Mataguayo family; Nivaĉle; Maká; Morphosyntax.
\end{abstract}

RESUMEN: El objetivo de este artículo es presentar y analizar esquemas de predicación nominal posesiva en nivaĉle y maká, dos lenguas chaqueñas de la familia mataguayo, con enfoque en el tipo sintético, que permite expresar, en una misma palabra, relaciones interpersonales del tipo soy tu hijo, muy escasamente documentadas en las lenguas del mundo. Para fines comparativos, se incluye también algunas observaciones sobre fenómenos similares en las otras lenguas de esta familia, el wichí/'weenhayek y el chorote.

Palabras Claves: Lenguas chaqueñas; Familia mataguayo; Nivaĉle; Maká; Morfosintaxis.

\section{Introducción}

Este trabajo describe un tipo de predicados que combina índices personales posesivos con nombres de parentesco o de relaciones interpersonales (esporádicamente otras, generalmente metafóricas). ${ }^{1}$ En muchas lenguas, las construcciones ecuativas de tipo $\mathrm{N}+$ $(\mathrm{COP})=(\mathrm{POS}-) \mathrm{N}($ Es $(\mathrm{mi})$ padre o Soy tu/un amigo) no presentan problemas particulares. Al contrario de lo que ocurre en nivaĉle y maká, y en las lenguas de la familia mataguayo en general, la presencia o ausencia de un índice posesivo solo tiene incidencia tangencial sobre la fórmula general. Siendo poco conspicuas, estas estructuras suelen subsumirse bajo el rubro 'ecuativo', y no pasan de ser un punto ciego en los estudios tipológicos de las lenguas. En nivaĉle y maká, que son lenguas con marcación de núcleo, el nombre poseído

\footnotetext{
${ }^{1}$ Quisiera dar constancia de mi sincero agradecimiento a los dos lectores anónimos por sus anotaciones y sugestiones que contribuyeron sustancialmente a mejorar una primera versión de este trabajo.
}

DOI: http://dx.doi.org/10.20396/liames.v20i0.8660913 
puede combinarse con dos índices, el que corresponde al sujeto de la construcción, y el otro con el poseedor, para formar un enunciado autónomo. Una única referencia (con dos ejemplos) a uno de los subtipos de esta construcción aparece en la gramática maká de Gerzenstein (1994: 163). Para el nivaĉle, menciono algunos ejemplos en mi gramática (Fabre 2016: 167-168), sin prestarles la debida atención. Hay menciones y ejemplos de esquemas predicativos con nombres de relaciones interpersonales en varios trabajos sobre la lengua mataguayo más hablada, el wichí/’weenhayek (§ 4.2.), pero que yo sepa, nunca han sido analizados de manera sistemática.

Fuera de las lenguas de la familia mataguayo, varias descripciones y estudios de lenguas particulares tratan de la predicación nominal posesiva con nombres de relaciones interpersonales. Estos estudios permiten apreciar que, si bien es sumamente raro, este fenómeno está documentado en unas pocas regiones del mundo, cuando menos en las Américas y en Australia. Amith \& Smith-Stark (1994) examinan tales construcciones en el nahuatl de Guerrero y las compara con datos de otras lenguas yutoaztecas, huichol, hopi, cora, y cahuilla. Para estas dos últimas lenguas, son imprescindibles los trabajos de Vázquez Soto (2013) y Seiler (1982). De suma importancia es también el artículo de Moser \& Marlett (1989) sobre el seri, lengua aislada de Sonora, y Halpern (1942), que examina aspectos gramaticales de los nombres de parentesco en yuma/quechan (familia cochima-yuman). Para otros datos esparcidos en descripciones de lenguas particulares, se pueden consultar los trabajos de Wolfart (1996: 403; 420-421) sobre el cree (algonquino), Troike (1996: 659-660) sobre el coahuilteco (lengua aislada), Mithun (1999: 253-254) sobre el tuscarora (iroqués), Chafe (2015: 100-110) para el seneca (iroqués) y Miller (1991; 2001: 84-85) con ilustraciones con verbos de parentesco en jamul tiipay/diegueño (familia yuman). Fenómenos que se encuadran perfectamente dentro de nuestra temática han sido documentados también en dos lenguas arawak habladas en Bolivia, el baure (Danielsen 2007: 181; 184; 193-195; 202; otro ejemplo en 2012: 325) y el mojeño trinitario (Rose 2018: 60). Esta rareza debería quizás ser matizada en la medida de que los ingredientes de este tipo de construcciones suelen encontrarse diseminados bajo diferentes acápites en las gramáticas, a la intersección del léxico (términos de parentesco, nombre alienables, nombres inalienables), la posesión adnominal ( $t u$ hijo), la posesión predicativa (tiene un hijo), la predicatividad verbal (incluso verbos de parentesco) y la predicatividad nominal, razón por la cual el fenómeno resulta difícil de detectar. Así, un estudio muy extenso como el de Queixalós (1998: 50 y 52; 2000: 304 y 306) sobre la lengua sikuani de los llanos de Colombia (familia guahibo) no dedica ningún sitio particular a la predicación con nombres de relaciones interpersonales. Si embargo, varios ejemplos incorporados en este trabajo demuestran sin lugar a duda la existencia de estructuras con nombres de parentesco provistos de dos índices que permiten expresar estas relaciones predicativas con una sola palabra.

Lamentablemente, no existe un término lingüístico único para referirse a las construcciones examinadas en este artículo. Obviamente, 'posesión predicativa' no conviene, puesto que remite a construcciones de otro tipo, cartografiadas, por ejemplo, en el trabajo de Stassen (2009). Halpern (1942), que trata las construcciones predicativas con nombre de relaciones interpersonales sin emplear el término, nota que 'kinship terms occur as verbs and as nouns'. Vázquez Soto (2013) habla de 'predicación de términos de parentesco', mientras que para Amith \& Smith-Stark (1994) son 'predicate nominal 
and transitive verbal expressions of interpersonal relations'. Hablar de 'relaciones interpersonales' tiene la ventaja de ser mucho menos restrictivo que 'términos de parentesco' o 'verbos (o nombres) de parentesco'. Por dicha razón, me parece apropiado para referirse a los tipos de predicados examinados en el presente trabajo, que, igual como en los verbos monotransitivos, exhiben dos índices personales. Amith \& Smith-Clark (1994) llaman 'relator' (REL) el lexema nominal central, cuya función es establecer la relación que rige entre los dos argumentos de la construcción. Adicionalmente, un índice posesivo va prefijado al relator. Resulta por otra parte claro que el índice de poseedor tiene implicancias semánticas distintas cuando aparece con un nombre como 'mesa': "MY FATHER is not one who is owned by me, but rather one who stands to me in a certain relation" (Sapir 1917). Pese a la ambigüidad del término, Amith \& Stark se refieren al sujeto como 'referente'. Como veremos, es probable que, tanto en nivaĉle como en maká, la presencia del sufijo aplicativo instrumental marca - en determinados casos - el término relacional como una entidad de tercera persona autónoma $(y o+t u+[\mathrm{ser}]$ madre-de).

En su gramática del maká, Gerzenstein (1994: 163) emplea el término 'predicación nominal posesiva' con referencia al tipo tratado aquí en $\S 3.2$, o sea una construcción sintética que consiste en un nombre provisto de dos índices personales (yo-tu-hijo $=$ soy tu hijo). Sin embargo, la autora ilustra también como casos de 'predicación nominal' cuatro ejemplos del tipo canónico $[\mathrm{POS}+\mathrm{N}]_{\mathrm{PRFD}}+\mathrm{FN}$ sín cópula y con sujeto de tercera persona (Gerzenstein 1994: 162; ver también aquí, ejemplos 21 y 22). En lo que sigue, considero como tipo de 'predicación nominal posesiva' toda construcción que establece una relación ecuativa entre un predicado nominal formado por un nombre poseído (POSEEDOR+POSEÍDO) y un referente humano que puede ser yuxtapuesto (frase nominal o pronombre libre - 21a-b y 22a-b), ir incluido en el predicado (representado por un índice personal o cero para la tercera persona - 23, 28), o ambos (37). Además, dos verbos (ser dueño § 3.5 y venir § 3.6) pueden emplearse en lugar del nombre, con índices de participantes verbales. Enunciados predicativos nominales posesivos que consisten en un solo nombre que lleva dos índices personales parecen sumamente raros. Un ejemplo de Rose (2018: 60) sugiere que el mojeño trinitario (Arawak) podría ser una de estas lenguas, pero la autora observa que si bien la frase nominal es opcional, está casi siempre presente. Obsérvese que los índices personales de (1a) pueden afijarse tanto a verbos como a nombres. Prefijado a un verbo, $p$ - está correferenciado con un sujeto de segunda persona singular. Prefijado a un nombre, señala un poseedor de segunda persona singular. Sufijado a un verbo, -un está coindexado con un objeto de primera persona singular, sufijado a un nombre, señala el sujeto de una predicación sobre una primera persona singular.

(1a) Mojeño trinitario (Rose 2018: 60)

nuti p-chicha-un

1SG.PRON 2sG-hijo-1sG

'Yo soy tu hijo'

Que yo sepa, en otras lenguas arawak de la zona, paresi-haliti (Brandão 2018: 92) o baure (Danielsen 2007: 193-194), el conominal no puede ser omitido.

Tanto el nivaĉle (con unos 16.350 hablantes en Paraguay y un estimado de algunos 500 hablantes en las provincias argentinas de Formosa y Salta) como el maká (1.500 
hablantes) son lenguas escasamente estudiadas. Para la primera lengua, se dispone de cuatro obras de cierta evergadura: la pequeña gramática escolar bilingüe de Seelwische (1975), la tesis doctoral de Stell (1989), que representa el primer estudio científico de la lengua, Fabre (2016), así como el recién salido trabajo de Campbell, Díaz \& Ángel (2020), que todavía no he podido consultar. Para el maká, la gramática pionera de Gerzenstein (1995) es la única fuente disponible, que aprofundiza detalladamente la fonología y la morfología de la lengua, pero aborda solo algunos aspectos de la sintaxis. ${ }^{2}$

Un rico caudal de datos del nivaĉle ha podido ser sido recogido por el autor durante el curso de tres trabajos de campo (junio/julio 2007, 2009 y 2011). ${ }^{3}$ Además, se han aprovechado varios textos publicados, entre los cuales destacan, en lo que hace a este estudio, Seelwische (1995) y la Biblia (SBP 1994). Para el maká, además de los imprescindibles trabajos de la añorada Ana Gerzenstein (1994 y 1999), gran pionera argentina de la lingüística chaqueña, la existencia de un vasto corpus de textos (el Nuevo Testamento) me ha permitido avanzar en el estudio de la lengua, llenando varias brechas tanto en el campo de la morfología como en el de la sintaxis. Pese a ser una traducción, el Nuevo Testamento resulta ser el único texto que cumula las ventajas de ser disponible, fiable, de notable extensión y aprovechable para un estudio lingüístico comparativo de las lenguas del grupo mataguayo (SBP 1994 para el nivaĉle, WBT 2013 para el maká, SBA 1997 para el chorote, SBA para el wichí y Claesson 1981-1996 para el 'weenhayek). Para la traducción de todos los ejemplos sacados de la Biblia, no se ofrece ninguna versión española particular (hay muchas), sino que se sigue de muy cerca, en la medida de lo posible, la versión vernácula.

Tras una presentación tipológica de los rasgos más relevantes de ambas lenguas para el tema del artículo ( $(1)$, se describen las diferentes construcciones ( $\$ 2$ y 3 ). Finalmente, para fines comparativos, se ofrece un breve panorama de construcciones similares en las dos otras lenguas de la familia mataguayo, el wichí/’weenhayek y el chorote (§4). termina con una conclusión general $(\S 5)$.

\section{Tipología general del nivaĉle y del maká}

El nivaĉle y el maká, junto con otras dos lenguas, wichí/'weenhayek y chorote, ${ }^{4}$ forman parte de la familia lingüística mataguayo, cuyos hablantes viven en la parte central de la región chaqueña, a ambos lados de las fronteras entre Bolivia, Argentina, y Paraguay, a lo largo de los dos ejes de los ríos Pilcomayo y Bermejo, desde los últimos contrafuertes andinos hasta su confluencia con el río de La Plata (ver nota 1 arriba). Tipológicamente estas lenguas pueden ser caracterizadas como (poli)sintéticas, con marcación radical de núcleo o, de acuerdo con la terminología de Mithun (2017: 63), de núcleo abierto. Ello

\footnotetext{
${ }^{2}$ Una lista de los trabajos sobre los pueblos y lenguas de la familia mataguayo es disponible en www.ling. fi/Entradas\%20diccionario/Dic=Mataguayo.pdf

${ }^{3}$ Por su paciencia e inagotable ánimo en orientarme en los arcanos de su nivaĉle nativo, quisiera agradecer a mi colaborador principal Félix Ramírez Flores.

${ }^{4}$ A diferencia del nivaĉle y el maká, el wichí/'weenhayek es una cadena dialectal dentro de la cual la intercomprensión entre hablantes de distintas variedades no siempre es cómoda. En cuanto al chorote, se han documentado tres variedades.
} 
significa que el núcleo verbal, que encierra, además de índices argumentales ${ }^{5}$ centrales y periféricos, una gama de aplicativos locativos, que sirven - entre otras funciones - para relacionar un argumento-figura sobre el fondo o la posición que le corresponde) así como un aplicativo instrumental. Un enunciado completo y gramatical puede estar conformado con una sola palabra, el núcleo verbal (1b) - (3). La primera línea de los ejemplos, que puede faltar en algunos datos del nivaĉle sacados de las notas del autor, aparece en una de las dos ortografías posibles, más o menos fonológica, de la fuente original mencionada después del nombre de la lengua que corresponde al ejemplo - "católica" para los ejemplos de Seelwische (y ocasionalmente en ejemplos sacados de mis notas de campo) y "mennonita" para los de la Biblia. Si no aparece mención alguna de fuente, se trata de un ejemplo nivaĉle sacado de los cuadernos de campo del autor de este artículo. La segunda línea, en itálicas, representa la segmentación morfológica de acuerdo con la grafía del AFI (Asociación Fonética Internacional/IPA), pero las vocales glotalizadas, que bajo circunstancias todavía por aclarar pueden perder su glotalización, van marcadas con una virgulita superpuesta (v̉). En el caso del maká, los ejemplos de Gerzenstein (1995 y 1999) aparecen en la grafía fonológica empleada por la autora.

(1b) Nivaĉle

$y i-f n^{3} y n-x-t y i \int a m$

3s-volar-INST-ARRIBA

'Se lo(s) lleva arriba' (comentario sobre un avión que despega, llevándose a un[os] niño[s])

(2) Nivaĉle

$x$-an-'akfi-xop

$1 \mathrm{~A}(3 \mathrm{P})$-poner-DEBAJO-ALREDEDOR

'Te lo voy a ceñir'
(3) Nivaĉle

$k^{2}-i s-e-f^{2} e$

$1 \mathrm{~A}(3 \mathrm{P})$-escribir-3-INST-EN

'Lo [= texto] anoto ahí [= cuaderno] con eso [= lápiz]'

Un rasgo de suma importancia en estas dos lenguas es la fluidez del paso de nombre a verbo o de verbo a nombre, razón por la cual nombre vs. verbo y referencialidad (FN) vs. predicatividad son parámetros inseparables para lograr una descripción satisfactoria. Todo nombre (4) o verbo (7) que aparece en una frase mínima precedido por un determinante es referencial y puede formar una frase nominal mínima. Todo nombre (5) o verbo (6) que aparece en una frase mínima no precedido por un determinante es predicativo y puede formar una frase verbal mínima (Tabla 1).

(4) Nivaĉle

ja nuu

$\left[\begin{array}{ll}x a & n u ̉\end{array}\right]_{\mathrm{REF}}$

$\mathrm{D}_{2}$-perro

'El/Un perro'
(5) Nivaĉle

nuu

$[\varnothing-n u ̉]_{\text {PRED }}$

3s-perro

'Es un/el perro'

${ }^{5}$ Empleo el término 'índice referencial' en el sentido de Haspelmath (2013), que considera el índice como un argumento propiamente dicho, con o sin conominal (frase nominal o pronombre). En el caso de las lenguas mataguayas, el conominal es opcional. 
(6) Maká (WBT)

t'ithayii

$\left[t^{\prime} \text {-ithayii }\right]_{\text {PRED }}$

3s-trabajar

'Trabaja'
(7) Maká (WBT) ${ }^{6}$

pa' t'ithayii

$\left[p-a^{2} \quad t^{3} \text {-ithayii }\right]_{\mathrm{REF}}$

$\mathrm{D}_{\mathrm{VI}}-\mathrm{M}$ 3s-trabajar

'(El/Un) trabajador (= el/uno que trabaja)'

Como se puede apreciar en la Tabla 1, una frase referencial mínima consiste en un lexema $\mathrm{N}$ o $\mathrm{V}$ precedido por un determinante, mientras un lexema $\mathrm{N}$ o $\mathrm{V}$ no precedido por un determinante forma una frase predicativa mínima. A este nivel de la representación, la morfología interna del lexema, sea verbal o nominal, no importa.

Tabla 1. Interrelaciones entre categorías lexicales, referencialidad y predicatividad en nivaĉle y maká

\begin{tabular}{|l|c|c|}
\cline { 2 - 3 } \multicolumn{1}{c|}{} & REFERENCIAL & PREDICATIVO \\
\hline $\mathrm{N}$ & {$[\mathrm{D}+\mathrm{N}]$} & {$[\mathrm{N}]$} \\
\hline $\mathrm{V}$ & {$[\mathrm{D}+\mathrm{V}]$} & {$[\mathrm{V}]$} \\
\hline
\end{tabular}

Hay dos clases principales de nombres propiamente dichos (en función referencial o predicativa): a) los que nunca pueden recibir prefijos posesivos (8), b) los que siempre deben ir precedidos por un prefijo posesivo (9-10) o, caso contrario, prefijo especial de poseedor desconocido o irrelevante (11). ${ }^{7}$ Algunos nombres que pertenecen a la primera subclase pueden usarse con un clasificador posesivo, uno de los cuales (clasificador posesivo general) es un prefijo al que se anteponen los prefijos posesivos (13). Existe también una serie de clasificadores especializados. Se trata de nombres obligatoriamente poseídos, que se anteponen o posponen al nombre principal desprovisto de marca posesiva (14-16a).

(8) Nivaĉle

pa lhaviim

pa tavim

D. $\mathrm{M}_{4}$ viento

'El/Un viento'

(12) Maká (WBT)

ke' loi

$k-e^{\text {? }} \quad$ t.o- $i$

$\mathrm{D}_{\mathrm{v}}$-PL 3POS.semilla-PL

'Sus semillas (de la planta)'
(10) Nivaĉle

lha lhôse

ta $\quad t-p s-e$

D.F 1 3Pos-hijo-F

'Su hija (de él/ella)'
(11) Nivaĉle

na vataôs

na vat-ans

D.M ${ }_{1}$ POS.IND-hijo

'El/un hijo (de fulano)'

(13) Maká (WBT)

ke' toqołoi

$k-e^{?} \quad$ to-qo-t.o-i

$\mathrm{D}_{\mathrm{v}}$-PL 3POS-CL.POS-3.POS.semilla-PL

'Sus semillas (del sembrador)'

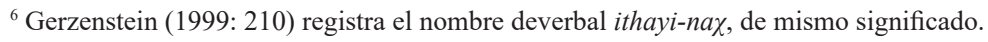

${ }^{7}$ Algunos nombres de parentesco obligatoriamente poseídos tienen un prefijo cero para la primera persona, idéntico a la forma empleada para dirigirse a la persona aludida. Gran parte de los nombres que denotan partes de un todo se usan solo con el prefijo de poseedor de tercera persona (12-13), que puede considerarse como lexicalizado con el nombre (en estos ejemplos separado de la base por un punto). Unos pocos nombres obligatoriamente poseídos tienen una marca cero para indicar un poseedor desconocido. 
(14) Maká (WBT)

ke' t'ithayii wittinhei

$k-e^{?} \quad t^{2}$-ithayii wit-tin-he-i

$\mathrm{D}_{\mathrm{v}}$-PL 3s-trabajar POS.IND-salvar/vivir.de-NMLZ.M-PL (= CL-POS.ANIM.DOM)

'Los esclavos (lit. los trabajadores-animales.domésticos machos de alguien)'

(15) Nivaĉle

$\begin{array}{ll}\text { ca yôc } & \text { yucuve } \\ k a \quad y-p k & \text { yukuve } \\ \text { D.M } \text { 1 }_{2} \text { POS-comida (= CL.POS.COMIDA) } & \text { pan } \\ \text { 'Mi pan (el pan que comí)' } & \end{array}$

(16a) Nivaĉle
ja yaje'
yiyeĉle
xa $y$-axe?
yiyekle
D.M 1 $_{2}$ POS-presa (= CL.POS.PRESA) tapir
'Mi tapir (la presa que maté)'

Los verbos nivaĉle y maká se reparten entre varias conjugaciones. Un verbo básicamente intransitivo prefija un índice personal sujeto (monovalente), mientras un verbo básicamente transitivo prefija dos índices personales, uno para el sujeto (agente) y otro para el objeto (paciente o recipiente según la rección de cada verbo particular - verbo con prefijo bivalente). De acuerdo con el alineamiento jerárquico $1>2>3$, el participante más elevado, sea sujeto u objeto, representa la pareja $\mathrm{A}+\mathrm{O}$, con la excepción de la combinación $2 \mathrm{~A}+1 \mathrm{O}$, donde ambos participantes aparecen. Existen varias estrategias para incrementar o disminuir la valencia. Cuando se agrega un sufijo causativo, el verbo cambia obligatoriamente de conjugación (-kumảx ${ }_{4}$ 'correr' $\rightarrow-k u m-t$ fat ${ }_{5}$ 'hacer correr' en 16b).
(16b) Nivaĉle
jacumaaj
xa-kumảx
1s-correr
'Corro'

\author{
(16c) Nivaĉle \\ jacumchat \\ xa-kum-tfat \\ $1 \mathrm{~A}(3 \mathrm{P})$-correr-CAUS \\ 'Lo hago correr'
}

(16d) Nivaĉle

tsicumchat

tsi-kum-tfat

(3A)1P-correr-CAUS

'Me hace correr'

La valencia de un verbo (intransitivo o transitivo) puede también incrementarse con un índice personal sufijado (llamados 'sufijos predicativos' en la parte inferior de la Tabla 5, pero ésta no es su única función), si bien esta opción es imposible con los sufijos causativos (16d). De acuerdo con la subcategorización del verbo, el sufijo puede ir solo o seguido por un aplicativo. Como veremos abajo, esto es frecuente en la predicación nominal posesiva. Las series de prefijos personales de las diferentes conjugaciones de los verbos básicamente intransitivos del maká aparecen en la tabla (2a), los del nivaĉle en la tabla (2b). Como se puede apreciar, la clase $\mathrm{C}_{0}$ del maká, que Gerzenstein considera prefijos de predicados nominales, corresponde a la serie 1 del nivaĉle. Las tablas (3a - maká) y (3b - nivaĉle) muestran las series de prefijos bivalentes en sus diferentes combinaciones. Para no complicar los cuadros, solo aparecen los principales alomorfos del realis. Los números romanos indican las series maká, los arábigos las del nivaĉle. Para el maká, V señala una 
FABRE - LA PREDICACIÓN NOMINAL POSESIVA...

vocal sujeta a la armonía vocálica (Gerzenstein \& Gualdieri 2003). Aparte de la primera persona inclusiva, los prefijos no indican número.

Tabla 2a. Prefijos monovalentes de los verbos maká (adaptado de Gerzenstein 1994)

\begin{tabular}{|l|c|c|c|c|c|c|}
\cline { 2 - 7 } \multicolumn{1}{c|}{} & $\mathrm{C}_{\mathrm{o}}$ & $\mathrm{C}_{\mathrm{I}}$ & $\mathrm{C}_{\mathrm{II}}$ & $\mathrm{C}_{\mathrm{III}}$ & $\mathrm{C}_{\mathrm{IV}}$ & $\mathrm{C}_{\mathrm{V}}$ \\
\hline 1s & $\mathrm{y}(\mathrm{V})-$ & $\mathrm{hVy}-$ & $\mathrm{h}(\mathrm{V})-$ & $\mathrm{ts}(\mathrm{i})-$ & $\mathrm{k}^{2}(\mathrm{i})-$ & $\mathrm{ha}-$ \\
\hline $2 \mathrm{~s}$ & $\mathrm{~V}-$ & $\mathrm{t}(\mathrm{V})-$ & $1 \mathrm{~V}-$ & $1 \mathrm{Vn}-$ & $1 \mathrm{~V}-$ & la- \\
\hline 3s & $\varnothing-$ & $\mathrm{t}(\mathrm{V})-$ & $\varnothing-$ & $\mathrm{n}(\mathrm{i})-\sim \mathrm{y}(\mathrm{i})-$ & $(\mathrm{i})-$ & (i)- \\
\hline 1INCL.s & $\mathrm{x}(\mathrm{V})-$ & $\mathrm{xit}(\mathrm{V})-$ & $\mathrm{xi}-$ & $\mathrm{xin}(\mathrm{i})-\sim \mathrm{xiy}(\mathrm{i})$ & $\mathrm{x}(\mathrm{i})-$ & $\mathrm{xu}-$ \\
\hline
\end{tabular}

Tabla 2b. Prefijos monovalentes de los verbos nivaĉle.

\begin{tabular}{|l|c|c|c|c|}
\cline { 2 - 5 } \multicolumn{1}{c|}{} & $\mathrm{C}_{1}$ & $\mathrm{C}_{2}$ & $\mathrm{C}_{3}$ & $\mathrm{C}_{4}$ \\
\hline $1 \mathrm{~s}$ & $\mathrm{ya}^{2}-$ & $\mathrm{xay}-$ & $\mathrm{ts}(\mathrm{i})-$ & $\mathrm{x}(\mathrm{a})-\sim \mathrm{k}^{2}(\mathrm{a})-$ \\
\hline $2 \mathrm{~s}$ & $\mathrm{a}^{2}-$ & lat- & $\mathrm{n}(\mathrm{a})-\sim \tan -$ & $\mathrm{t}(\mathrm{a})-$ \\
\hline $3 \mathrm{~s}$ & $\varnothing-$ & $\mathrm{t}(\mathrm{a})-$ & $\mathrm{n}(\mathrm{i})-\sim \mathrm{y}(\mathrm{i})-$ & $\mathrm{y}(\mathrm{i})-$ \\
\hline 1INCL.s & kas- & $\int \mathrm{t}(\mathrm{a})-$ & $\int \mathrm{t}(\mathrm{a})-\sim \int \tan -$ & $\int \mathrm{t}(\mathrm{a})-$ \\
\hline
\end{tabular}

Tabla 3a. Prefijos bivalentes de los verbos maká (adaptado de Gerzenstein 1994)

\begin{tabular}{|c|c|c|c|}
\hline & $\mathrm{C}_{\mathrm{VI}}$ & $\mathrm{C}_{\mathrm{VI}}$ & $\mathrm{C}_{\mathrm{VII}}$ \\
\hline $1 \mathrm{~s}+2 \mathrm{o}$ & \multicolumn{3}{|c|}{$k^{2}(V)-$} \\
\hline $2 s+3 o$ & $h(i)-$ & $\mathrm{hVn}-$ & $\mathrm{hV}-$ \\
\hline $3 s+10$ & \multicolumn{3}{|c|}{ ts(i)- } \\
\hline $2 s+10$ & \multicolumn{3}{|c|}{ 1V-ts(i)- 1V-s- } \\
\hline $2 s+3 o$ & 1(i)- & IVn- & $1(V)-$ \\
\hline $3 \mathrm{~s}+2 \mathrm{O}$ & \multicolumn{3}{|c|}{$n(V)-$} \\
\hline $3 \mathrm{~s}+3 \mathrm{o}$ & $y(i)-$ & $n(i)-$ & $\varnothing-$ \\
\hline 1INCL.S +30 & \multicolumn{3}{|c|}{ xit(V)- } \\
\hline $3 \mathrm{~s}+1 \mathrm{INCL} .0$ & \multicolumn{3}{|c|}{ xin(i)- } \\
\hline
\end{tabular}


Tabla 3b. Prefijos bivalentes de los verbos nivaĉle

\begin{tabular}{|l|c|}
\cline { 2 - 2 } \multicolumn{1}{c|}{} & $\mathrm{C}_{5}$ \\
\hline $1 \mathrm{~s}+2 \mathrm{o}$ & $\mathrm{k}^{2}(\mathrm{a})-$ \\
\hline $2 \mathrm{~s}+3 \mathrm{o}$ & $\mathrm{x}(\mathrm{a})-$ \\
\hline $3 \mathrm{~s}+10$ & $\mathrm{ts}(\mathrm{i})-$ \\
\hline $2 \mathrm{~s}+10$ & $\mathrm{1}-\mathrm{ts}(\mathrm{i})-\sim$ ta-s- \\
\hline $2 \mathrm{~s}+3 \mathrm{o}$ & $\mathrm{t}(\mathrm{a})-$ \\
\hline $3 \mathrm{~s}+2 \mathrm{o}$ & $\mathrm{n}(\mathrm{a})-$ \\
\hline $3 \mathrm{~s}+3 \mathrm{o}$ & $\mathrm{y}(\mathrm{i})-$ \\
\hline $1 \mathrm{INCL} . \mathrm{s}+3 \mathrm{O}$ & $\int \mathrm{t}(\mathrm{a})-$ \\
\hline $3 \mathrm{~s}+1 \mathrm{INCL} . \mathrm{O}$ & $\int \mathrm{jin}-$ \\
\hline
\end{tabular}

(16e-f) del maká ilustran el uso de verbos básicamente intransitivos que incrementan su valencia con objetos sufijados. El verbo nivaĉle de (16g) es monotransitivo. El prefijo del verbo -tis s 'dar (a alguien), de acuerdo con su subcategorización, autoriza un agente y un objeto recipiente. La presencia de un paciente necesita agregar índice personal seguido por el aplicativo instrumental. En esta construcción, el verbo funciona como ditransitivo. ${ }^{8}$

(16e) Maká (Gerzenstein 1999: 349)

k'itsowol'aXix na' mats

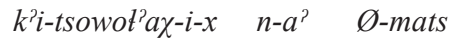

1s-soñar-3-INST $\quad \mathrm{D}_{\mathrm{II}}$-M $\quad$ 1POS-hermano.mayor

'Sueño/Soñé con mi hermano' (-tsowot'a $\chi_{\text {iv }}$ 'soñar')

(16f) Maká (Gerzenstein 1999: 115)

lanak'esyik'wi

tan-ak'es-yi-k'wi

2s-separarse/estar.separado-1-sEP

'Te separaste de mí' (-ak'es ${ }_{\mathrm{iii}}$ 'separarse; estar separado')

(16g) Nivaĉle (Seelwische 1995: 106)

$\begin{array}{llll}\text { jatisesh } & \text { jayu } & \text { pava } & \text { vat'uijatshiyis } \\ \text { xa-tis-e-f } & \text { xayu } & \text { pa-va } & \text { vat'-ui-xat-fi-yis } \\ \text { 1s(3o)-dar.a-3-INST } & \text { PROSP } & \text { D }_{4} \text { PL.NO.HUM } & \text { POS.IND-entrar-NMLZ-EN-PL }\end{array}$

'Les voy a regalar ropas (lit. algo en que meterse)'

${ }^{8}$ Existe un sinónimo de 'dar (monotransitivo)', -xut ${ }_{5}$, donde el prefijo representa un sujeto agente con objeto paciente. Para introducir el recipiente, se debe agregar un sufijo de persona seguido ya por el aplicativo locativo distal, ya por el benefactivo. 


\section{Predicación nominal con términos de relaciones interpersonales en nivaĉle y maká}

Las bases nominales tienen flexión personal, si bien con ciertas restricciones pragmáticas y semánticas. El paradigma de los índices personales consta de un sistema tres índices, más uno de primera persona inclusiva, el único miembro abiertamente marcado como plural. ${ }^{9}$ Los índices personales posesivos y predicativos prefijados no pueden combinarse con una misma base, si bien en maká, el prefijo de poseedor indefinido puede ir precedido por un índice predicativo de persona [último ejemplo de la columna derecha de (17)].

Tabla 4. Índices personales posesivos y predicativos de los nombres nivaĉle y maká ( $\mathrm{V}=$ vocal determinada por la harmonía vocálica)

\begin{tabular}{|c|c|c|c|c|c|c|}
\hline & & & & & \\
\hline & & 1 & 2 & 3 & $1 \mathrm{INCL}$ & INDEF \\
\hline \multirow{2}{*}{$\begin{array}{l}\text { PREFIJO } \\
\text { POSESIVO }\end{array}$} & MAKÁ & $y(i)-$ & (V)- & $1(V)-$ & in- & wVt- \\
\hline & NIVAĈLE & $y(i)-$ & (a)- & $1(V)-$ & kas- $\sim$ kats(i) & wat(a)- \\
\hline \multirow{2}{*}{$\begin{array}{l}\text { PREFIJO } \\
\text { PREDICATIVO }\end{array}$} & MAKÁ & $y(V)-$ & V- & $\varnothing-$ & $x(V)-$ & \\
\hline & NivaĈLE & $\mathrm{ya}^{\text {?- }}$ & $a^{2}-$ & $\begin{array}{l}\varnothing- \\
\left(\sim-\mathrm{e}-\int\right)\end{array}$ & kas- kats(i)- & \\
\hline SuFIJO & MAKÁ & -ye & $-\mathrm{e}$ & $-\mathrm{i} \sim-\mathrm{V}$ & -in & \\
\hline PREDICATIVO & NivaĈLE & $\begin{array}{l}\text {-yi } \\
\text {-ya }(+ \text { BEN })\end{array}$ & $-2 a$ & $\begin{array}{l}-\mathrm{e} \\
-\mathrm{ta} \\
-\varnothing\end{array}$ & $\begin{array}{l}\text {-xỏ } \\
\text {-katsi- }\end{array}$ & \\
\hline
\end{tabular}

Solo los nombres no poseíbles pueden emplearse con los índices argumentales predicativos prefijados (17). En nivaĉle, la tercera persona puede opcionalmente marcarse con un sufijo de tercera persona seguido por el aplicativo instrumental (-e-f), en cuyo caso el significado puede variar entre 'es (un/una)X' o 'se transforma/tiene la apariencia exterior de (un/una) X’ (18a).

(17) Nivaĉle Maká (Gerzenstein 1994: 158)

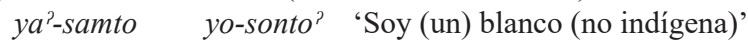

$a^{\text {'-samto }} \quad$ o-sonto? 'Sos (un) blanco'

Ø-samto $\quad$-sonto? '(Él) es (un) blanco'

kas-samto xo-sonto? 'Nosotros (incl.) somos blancos'

ye-wit-qatxat-en-ek 'Soy cocinero' (Gerzenstein 1999: 303)

1s-POS.IND-cocinar-CAUS-NMLZ

${ }^{9}$ El plural de sujeto (también puede ir coindexado con el objeto cuando si se refiere a un índice personal sufijado) o poseedor va marcado con un sufijo de plural coordinativo. Así, por ejemplo, el plural (exclusivo) de primera persona se puede parafrasear como 'Voy al cine contigo', o, si el hablante quiere emplear el pronombre sujeto (opcional), 'Nosotros, voy al cine contigo'. 
(18a) Nivaĉle

Ø-yonis 'Es/Era un zorro' Ø-yonis-e-f'Es/Era un zorro' 'Tiene/Tenía la apariencia de un zorro' 'Se transforma/transformó en un zorro'

Tengo registrado un solo caso donde el nivaĉle permite la prefijación de una primera persona sujeto predicativo con poseedor de tercera (18b). Nótese la presencia del sufijo verbal de movimiento asociado de venida anticipada de participante no sujeto ( $k^{\text {'oya }}$, coindexado con las ovejas). Son bastante frecuentes, por lo menos en los textos bíblicos, las extensiones metafóricas del uso de los predicados de relaciones interpersonales.

(18b) Nivaĉle (SBP 1994, Juan 10:7)

\begin{tabular}{|c|c|c|c|c|}
\hline yivaatsha & ya lhech & $\mathrm{ti}$ & & \\
\hline$y i$-vảtfa & $y a^{2}-$ tet & $t i$ & & \\
\hline $\begin{array}{l}\text { 1-PRON } \\
\text { ya lhashieshc'ova }\end{array}$ & 1-PRON.ENF & $\mathrm{SUB}_{1}$ & nava & tashinshtas \\
\hline$y a^{2}-t-a j i-e-f-k^{2} o y a$ & & & $n a-v a$ & tafinfta-s \\
\hline 1s-3Pos-boca/puert & INST-VENT.ANT & & $\mathrm{D}_{1}$-PL.NO.HUM & oveja-PL \\
\hline
\end{tabular}

'Soy yo quien soy la puerta para (esperar que entren) estas ovejas'

En (18c) y (18d), el prefijo va seguido por una forma especial del posesivo de tercera persona (normalmente $t a-\sim t$-), tav-, que bloquea la permutación con los otros prefijos posesivos. Hasta cierto punto, se podría traducir lavk'itxen-ax como 'salva-dor', donde el posesivo de tercera persona formaría parte del nombre (pragmáticamente, 'salvador' y 'salvado' son inseparables). Hunt (1940: 106 - redactado antes de 1929) señala un fenómeno similar en wichí (mataco), donde un índice de tercera persona le- aparece en una función que dicho autor asimila a un simple 'artículo', incluso cuando sigue a dos índices (o-a-le-chen-ek [1-2-ART-enviar-PRT] 'eres mi enviado') (v. también 4.2. abajo y ej. 88-89). Sin los pronombres enfáticos (opcionales), la lectura no marcada de la segunda palabra sería 'Eres mi salvador' en (18c) y 'Soy tu salvador' en (18d).

Como se puede apreciar, el sufijo de segunda (18c) o primera (18d) persona representa el sujeto de la predicación (v. tipo 3.3.d abajo). La presencia de los pronombres acarrea sin embargo un cambio radical, puesto que ambos se vuelven sinónimos. Evans (2000: 120121) reporta un fenómeno similar en verbos de parentesco de dos lenguas australianas, el ilgar y el iwaidja, y considera la orientación a uno u otro participante representa un cambio de pivote. Siguiendo este mismo razonamiento, (18c) debería analizarse como una estructura relativa con núcleo interno del tipo descrito en Fabre (2016: 389-391), donde el nombre predicativo de relación interpersonal aparece como calco de una citación directa. Sin bien (18d) tiene una estructura superficial idéntica, representa la fórmula no marcada, con el pronombre libre coindexado con el sufijo sujeto de primera persona.

(18c) Nivaĉle (SBP, Salmo 35: 3)

a'lhech yi'lhavqu'ilhjenaj'ash

$a^{2}$-tet $\quad y i-t a v-k^{2}$-itxen-ax-'a- $\int$

2PRON-ENF 1POS-DESP-MED.POS-salvar-NMLZ-2-INST

'Tú eres mi salvador' (lit. eres tú [el que (dice)] eres mi salvador') 
(18d) Nivaĉle (SBP, Isaías 60: 16)

ya'lhech a'lhavqu'ilhjenajyish

ya'-tet a $\quad a^{2}-t a v-k^{2}-i t x e n-a x-y i-\int$

1PRON-ENF 2POS-DESP-MED.POS-salvar-NMLZ-1-INST

'Tú eres mi salvador' (lit. soy yo, soy tu salvador)

(18e) ilustra el mismo fenómeno que (18c), pero ‘yerno’ está subordinado al verbo ‘decir’.

(18e) Nivaĉle (SBP, 1 Samuel 18: 21)

\begin{tabular}{|c|c|c|c|}
\hline ueesh & lhtees & ca & atômit'aayish \\
\hline $10-k$ & t-tẻs & $k a$ & $a-$ tpmit $^{2} a-a-y i-\int$ \\
\hline . $\mathrm{M}_{1}$-DEM-3-INST & 3s-decir & $\mathrm{SUB}_{2}$ & 2POS-yerno-IRR-1-INST \\
\hline
\end{tabular}

'Ahora dirás (podrás decir) que eres mi yerno (lit. yo soy tu yerno)'

(18f) es un ejemplo similar a la lectura (plural) no marcada de (18d) desprovista del pronombre libre (soy tu salvador/el salvador de ustedes), con dos variaciones: el prefijo es predicativo en lugar del posesivo en (18c-d) y el sufijo de segunda persona seguido por el benefactivo funciona como recipiente de la actividad.

(18f) Nivaĉle (SBP, Isaías 41: 14)

yalhavqu'ilhjenajelh'am

$y a-$ tav-k'-itxen-ax-et ${ }_{i}{ }^{2} a_{i}-m$

1S-DESP-MED.POS-salvar-NMLZ-PL-2-BEN

'Soy salvador de (para) ustedes'

Sin embargo, las construcciones con sufijo instrumental son mucho más comunes (18c-e) y $(18 \mathrm{~g}-\mathrm{j})$.

(18g) Nivaĉle (SBP, Salmo 31: 3)

yi'utej'ash

yi-?utex-'a- $\int$

1POS-piedra-2-INST

'Eres mi roca'

(18h) Nivaĉle (SBP, Salmo 71: 3)

a'utejesh

$a^{2}$-utex-e- $\int$

2POS-piedra-3-INST

'Eres (una) roca'

(18i) Nivaĉle (SBP, Isaías 44: 21)

yicavo'ash

yi-kavo- ${ }^{2} a-f$

1pos-siervo-2-inst

'Eres mi siervo'

(18j) Nivaĉle (SBP, Jeremías 51: 20)

a'lhech yitôjôyech'ash

$a^{2}$-tet $\quad y i$-tpxpyets-'a- $\int$

2-pron.enf 1pos-maza-2-inst

Tu eres mi maza'

Los prefijos posesivos y predicativos no pueden combinarse en una misma base (19) y (20). Para expresar estas configuraciones, hay que recurrir a las construcciones listadas a continuación. 
(19) Nivaĉle

*ya'-t-tata

1s-3Pos-padre

'Soy padre de él/ella'
(20) Maká

*ya-ta-tata

1s-3Pos-padre

'Soy padre de él/ella'

Tanto el nivaĉle como el maká presentan en combinaciones con un sujeto de tercera persona dentro de una frase nominal (parte referencial/tópico) y un predicado/comentario que consiste en un prefijo de poseedor y el nombre (21a-b). Las construcciones presentativas consisten en un presentativo seguido nombre poseído (22a-b).

(21) Maká (Gerzenstein 1999 - segmentación y glosas del autor)

(21a)

\begin{tabular}{|c|c|c|}
\hline yixuwayka' & na' & Martín \\
\hline$\left[y i-x u w a y k a^{?}\right]_{\mathrm{PRED}}$ & {$\left[n-a^{?}\right.$} & Martín $]_{\mathrm{REF}}$ \\
\hline $\begin{array}{l}\text { 1Pos-amigo } \\
\text { Pi }\end{array}$ & $\mathrm{D}_{\mathrm{II}}-\mathrm{M}$ & Martín \\
\hline
\end{tabular}

(21b)

$$
\begin{array}{lll}
\text { yikifeki' } & \text { ne' } & \text { Juana } \\
{[y i-k i-f e-k i ']_{\mathrm{PRED}}{ }^{10}} & {\left[n-e^{?}\right.} & \text { Juana }]_{\mathrm{REF}} \\
\text { 1POS- ?-CMP-F } & \mathrm{D}_{\mathrm{II}}-\mathrm{F} & \text { Juana } \\
\text { 'Mi vecina es Juana' } & \\
(\sim \text { '[La] Juana es mi vecina') }
\end{array}
$$

(22a) Nivaĉle (Seelwische 1995: 146)

$\begin{array}{llll}\text { navôque } & \text { lheiyis } & \text { cava } & \text { lhavtsatis } \\ n a-v p-k e & t \text {-ei-yis } & k a \text {-va } & \text { ta-vtsat-is } \\ \mathrm{D}_{1} \text {-PL.NO.HUM-DEM } & \text { 3POS-nombre-PL } & \mathrm{D}_{3} \text {-PL.NO.HUM } & \text { 3POS-aldea-PL } \\ \text { 'Estos son los (sus) nombres de sus aldeas' } & \end{array}$

(22b) (Seelwische 1995: 204)

nôque aôs anôque amimi

nn-ke $\quad$ Ø-ans, a-nn-ke a-mimi

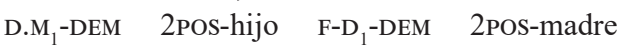

'Éste es tu hijo, ésta es tu madre'

\section{Predicación nominal posesiva con nombres de relaciones interpersonales}

La gramática maká de Gerzenstein (1995: 163) contiene dos ejemplos, (23) y (24), ${ }^{11}$ que identifica como tipos de predicación nominal posesiva. La autora llama la atención sobre las particularidades siguientes: cuando el prefijo $k^{\prime} e^{\prime}$ - se une a un verbo (25), representa la combinación de dos índices argumentales, primera persona sujeto y una segunda objeto. Por su parte, el segundo índice personal de (23), yi-, es posesivo (cf. 26);

${ }^{10}$ La base $-k i(\sim$ nivaĉle $t i)$ no está atestada aisladamente en maká. El cognado nivaĉle yi-tfi-fa-tfe (igual segmentación que el ejemplo maká) 'Es mi amiga' está formado de una manera transparente sobre la partícula coordinativa $t i j$ 'y'.

${ }^{11}$ Gerzenstein (1999) ofrece diez ejemplos de la combinación de primera persona sujeto y poseedor de segunda persona ( $\left.k^{\prime} e-y i-\sim k^{\prime} e-y e-\sim k^{\prime} a-y-\right)$ 'soy tu $\mathrm{X}$ ' y dos de la de segunda persona sujeto y poseedor de primera (e-ts- $\sim e-s-)$ 'eres mi X'. Para fines comparativos, he modificado la glosa original del ejemplo (25) de Gerzenstein: 1/2-amar. 
en (24), ambos prefijos son verbales: $e$ - ' $2^{\mathrm{a}} \mathrm{p}$.' (sujeto) $+t s$ - ' $1^{\mathrm{a}} \mathrm{p}$.' (objeto) como se puede observar comparando con (27). La primera línea de las glosas de (23) y (24) se reproduce como en original de Gerzentein (a). Para fines comparativos, mi propio análisis aparece en la segunda línea (b).

(23) $\left[k^{\prime} e-y i \text {-tata }\right]_{\text {pred }}$

(a) 2-1-padre

(b) $1 \mathrm{~s}(2 \mathrm{o})-1$ pos-padre 'Soy tu padre'

(25) $\left[k^{\prime} e-s u^{\prime} u n\right]_{\text {pred }}$ $1 \mathrm{~s}(2 \mathrm{o})$-amar 'Te amo'

(27) $[\text { e-tsi-fen }]_{\text {pred }}$ 2s-1o-ayudar '¡Ayúdame!'

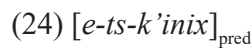

(a) 2-1-hermano.menor

(b) 2s-1o-hermano.menor

'Eres mi hermano menor'

(26) $\left[n-a^{\prime} \quad y i \text {-tata }\right]_{\mathrm{ref}}$ $\mathrm{d}_{\mathrm{ii}}-\mathrm{m} \quad$ 1 Pos-padre 'Mi padre'

Pese a pequeñas confusiones debidas al escaso número de datos, ${ }^{12}$ Gerzenstein nos presenta construcciones lo suficientemente inhabituales como para despertar nuestro interés.

En el sistema común de cuatro personas (1,2,3,1 INCL), se dan nueve configuraciones posibles. Las formas reflexivas no son gramaticales. ${ }^{13}$ En la combinación $3+3$, los participantes deben ser distintos.

${ }^{12}$ El prefijo $k$ 'e- de (23) debe analizarse como en (25): contiene dos índices argumentales (1s/20+1 Pos). No se explica porque el primer prefijo (segunda persona e-) de (24) lleva la forma de irrealis (el realis sería te-). La traducción de (24) debería ser '(que) seas mi hermano menor'. Supongo que la confusión se haya presentado en una situación de elicitación, si bien el diccionario de Gerzenstein (1999) registra un ejemplo adicional con esta misma secuencia $e-t s-$.

${ }^{13} \mathrm{El}$ 'weenhayek (y talvez otras variedades del wichí) es la única lengua del grupo mataguayo que permite formas reflexivas, por lo menos con sujetos de primera o tercera persona de algunos nombres. Sin embargo, en ambos casos el nombre de relación interpersonal lleva una marca de tercera persona. Los dos ejemplos de (90) en 4.2. pueden ser parafraseados respectivamente como "soy su propio dueño" y "ellos son sus propios dueños". 
Tabla 5. Combinaciones de índices personales en la predicación nominal posesiva con nombres de relaciones interpersonales en nivaĉle y maká. Los números en las celdas remiten a las secciones correspondientes del artículo. azul $=$ solo en nivaĉle, rojo $=$ solo en maká, $\sin$ color $=$ en ambas lenguas. Nótese que una misma configuración puede expresarse con estructuras alternativas.

\begin{tabular}{|c|c|c|c|c|}
\hline \multirow[t]{2}{*}{ POSEEDOR } & \multicolumn{4}{|c|}{ SUJETO DE LA PREDICACIÓN } \\
\hline & 1 & 2 & 3 & 1INCL \\
\hline 1 & & $\begin{array}{l}\text { 3.2.a; 3.2.c; 3.2.d; } \\
\text { 3.3.d; 3.5; 3.6. }\end{array}$ & $\begin{array}{l}\text { 3.1.a; 3.3.e; } 3.5 \text {; } \\
\text { 3.6. }\end{array}$ & \\
\hline 2 & 3.3.c; $3.5 ; 3.6$. & & $\begin{array}{l}\text { 3.1.b; 3.3.b; 3.3.f; } \\
\text { 3.5.; 3.6. }\end{array}$ & \\
\hline 3 & 3.3.a; 3.4; 3.5; 3.6. & 3.3.b; $3.4 ; 3.5 ; 3.6$ & 3.1.c; 3.5; 3.6. & $\begin{array}{l}\text { 3.3.g; } 3.4 ; 3.5 \text {; } \\
\text { 3.6. }\end{array}$ \\
\hline 1INCL & $S<$ & $M$ & 3.1.d & \\
\hline
\end{tabular}

Obsérvese que los conominales (pronombres o frases nominales/determinativas) son opcionales. La lista de combinaciones que sigue se ha organizado tomando como punto de partida el sujeto de la predicación, a partir del esquema más sencillo.

3.1. El tipo más sencillo combina un sujeto de tercera persona con cualquier índice posesivo. La fórmula es [POS-N $]_{\text {PRED }}$. Puede ir acompañado por un conominal (FD o PRON), pero éste es opcional. La fórmula básica sola (en negritas) forma un enunciado gramatical. Opcionalmente, el nivaĉle puede marcar el sujeto con el sufijo de tercera persona seguido por el aplicativo instrumental.

a) Poseedor de primera persona. Alternativamente, la misma configuración puede expresarse con la construcción 3.3.d, 3.5. y 3.6. Para el maká, se da una opción más, 3.3.e.

(28) Nivaĉle

yija'ya

yi-xa'ya

1Pos-cónyuge

'Es mi cónyuge'

(29) Maká (WBT, Juan 8: 39)

Pa' Abraham'ik'i hik pakha' tata'al

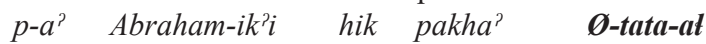

$\mathrm{D}_{\mathrm{VI}}-\mathrm{M} \quad$ Abraham-ENF ${ }^{14}$ ENF $\mathrm{DM}_{\mathrm{VI}} 3$ PRON 1 POS-padre-PL

'Abraham es nuestro (excl.) padre'

${ }^{14}$ No se ha podido determinar la función exacta del marcador $-V k i$, que se sufija a verbos y nombres. Como se puede apreciar al comparar varios enunciados idénticos que se repiten a lo largo del Nuevo Testamento, no pasa de ser opcional. Se trata posiblemente de un focalizador. Ha sido tentativamente glosado ENF. 
(30) Maká (WBT, Juan 9: 20)

tsikfe'li'łets

ts-ikfel-it-ets

in

in

nakhaa'ija

hakha'

ya'si'1

1s-saber-PL.COORD-OBJ SUB

qa $\varnothing$-ta't-i-x-xi'-texem

nakhaa-ixa

hakha?

y-as-it

y 3s-venir-3-INST-EN-DENTRO

$\mathrm{D}_{2}$ M.3.PRON-ENF

$D_{\text {IV }}$ M.3PRON

1POS-hijo-PL.COORD

wit-tawex

in

Ø-puk'al-tax

'Sabemos que éste es nuestro hijo, y que nació (lit. vino desde dentro del vientre) ciego'

b) Poseedor de segunda persona (únicamente en maká). Alternativamente, la misma configuración puede expresarse con la construcción 3.3.b, también exclusiva del maká, o 3.5. y 3.6., opciones válidas para las dos lenguas.

(31) Maká (WBT, Juan 8:39)

$\begin{array}{lll}\text { atatayi'it } & \text { kakha' } & \text { Abraham'ik'i } \\ \text { a-tata-yit } & k a k h{ }^{\text {? }} & \text { Abraham-ik'i } \\ \text { 2POS-padre-PL.COORD } & \text { DM }_{\mathrm{v}-} \text { 3PRON } & \text { Abraham-ENF }\end{array}$

'Abraham es el padre de ustedes'

(32) Maká (WBT, Juan 4: 18)

$\begin{array}{lllll}\text { hakha' } & \text { hane'ej } & \text { in'ejup } & \text { nite' } & \text { ewhe'ye' } \\ \text { hakha' } & \text { hane'ex } & \text { i-n-e-xup } & \text { nite? } & \text { Ø-ewheye } \\ \mathrm{D}_{\mathrm{IV}} .3 \mathrm{PRON} & \text { ahora } & \text { 3s-estar-2-JUNTO } & \text { NEG } & \text { 2POS-esposo }\end{array}$

'El que ahora está contigo no es tu marido'

c) Poseedor de tercera persona (siempre distinto del sujeto). En (34a), el nivaĉle lleva el sufijo (sujeto) opcional de tercera persona seguido por el aplicativo instrumental. Alternativamente, la misma configuración puede expresarse con la construcción 3.5. o 3.6.

(33) Nivaĉle

lhaôs

t-ans

3POs-hijo

'Es hijo de ella/él' (34a) Nivaĉle (SBP, Hebreos 5: 8)

yamsha ti Lhô'sesh pa Dios

yamfa ti $\boldsymbol{t}$-ns-e-f pa Dios

aunque SUB $_{1}$ 3Pos-hijo-3-INST $\quad$ D.M $_{4}$ Dios

'Aunque era hijo de Dios'

(34b) Maká (WBT, Hebreos 5: 8)

yemjeetax in la'staxija pa' Dios

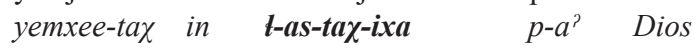

aunque-CON SUB 1 3POS-hijo-CON-INT $\mathrm{D}_{\mathrm{v}}-\mathrm{M}$ Dios

'Aunque era hijo de Dios'

d) Poseedor de primera persona inclusiva (única opción para ambas lenguas)

(35) Nivaĉle

casch'inish

kas-ty'inif

1INCL.POS-hermano.menor

'Es nuestro hermano menor' 
(36) Maká (WBT, Juan 8:54)

$\begin{array}{llll}\text { pakha'an } & \text { hik } & \text { pakha' } & \text { inqe Dios } \\ \text { pakha-'an } & \text { hik } & \text { pakha' } & \text { in-qe-dios } \\ \text { DM }_{\mathrm{vI}} \text { 3PRON-ENF } & \text { ENF } & \text { DM }_{\mathrm{VI}} \text { 3PRON } & \text { 1INCL-CL.POS-dios } \\ \text { 'Él es nuestro Dios' } & & \end{array}$

3.2. Poseedor de primera persona con sujeto de segunda. Se han identificado cuatro construcciones, en dos de las cuales el nombre va precedido por el mismo índice personal $1+2$ que se prefija a verbos transitivos, $1 \mathrm{~S}(>2 \mathrm{O})$. En ambos casos, el alineamiento jerárquico $1>2>3$ selecciona el participante más alto, cualquiera sea su papel semántico, sujeto u objeto, para saturar el prefijo de una base transitiva. ${ }^{15}$ Obsérvese que, con una base transitiva, el prefijo siempre implica dos participantes. Para agilizar la lectura de los ejemplos presentados aquí, el participante rechazado aparece entre paréntesis. La única excepción a la regla es cuando una segunda persona sujeto se combina con una primera objecto, en cuyo caso ambos índices van prefijados. Ahora bien, como se puede observar en la Tabla 6, el papel de los participantes cambia drásticamente cuando se unen a una base nominal.

Tabla 6. Función de los índices prefijados de primera y segunda persona con base verbal y base nominal.

\begin{tabular}{|l|c|c|}
\cline { 2 - 3 } \multicolumn{1}{c|}{} & \multicolumn{2}{c|}{ FUNCIÓN DE LOS ÍNDICES PERSONALES PREFIJADOS } \\
\cline { 2 - 3 } \multicolumn{1}{c|}{} & $1^{\mathrm{a}}$ PERSONA & $2^{\mathrm{a}}$ PERSONA \\
\hline BASE VERBAL & Sujeto & Objeto \\
\hline BASE NOMINAL & Poseedor & Sujeto \\
\hline
\end{tabular}

La diferencia entre la construcción del maká y la del nivaĉle resalta en el tratamiento de la indexación del poseedor. En el maká, como ya Gerzenstein (1994: 163), el prefijo posesivo de primera persona aparece entre el prefijo verbal y la base nominal. Este prefijo posesivo no obligatorio es redundante, puesto que el prefijo verbal es bivalente (38a) y (38b). El nivaĉle presenta otra forma redundante (37), que aparece junto con el sufijo de segunda persona instrumental. En nivaĉle, el índice personal seguido por el aplicativo instrumental es frecuente, ente otras funciones, como marca de objeto de muchos verbos básicamente intransitivos.

a) $[1 \operatorname{POS}(2 \mathrm{~s})-\mathrm{N}]_{\mathrm{PRF}}$. Solo documentado en nivaĉle. Las variantes con sufijo de segunda persona + aplicativo instrumental (reduplicación del índice ya presente en el prefijo) (37a) y (37b) o tercera persona + aplicativo instrumental (índice que representa el nombre poseído) (39) no pasan de ser opcionales. Se pueden contrastar con los ejemplos no marcados (sin

${ }^{15}$ La saturación mínima de la posición prefijada de una base transitiva incluye dos participantes, sujeto (agente) y objeto (paciente o recipiente - opción lexicalmente determinada). Se trata, pues, de una posición transitiva monovalente. Cualquier participante adicional, aumento de valencia o marcación de plural, va sufijada. El conominal (nombre o pronombre) coindexado con el índice verbal es opcional, pero su omisión implica una relación anafórica. Puesto que una base verbal solo puede ser transitiva o intransitiva, el hablante que quiere omitir toda referencia al objeto debe emplear una base transitiva, mediante derivación morfológica (antipasivo), o recurrir a un verbo supletivo. 
redundancia) (37c) y (37d). Como se trata del alineamiento jerárquico (cf. arriba y nota 9), solo el índice de primera persona aparece, pero simultáneamente representa la segunda persona latente. Recuérdese que también los conominales (frases nominales y pronombres libres) son opcionales. Puede alternar con 3.2.d, 3.3.d (solo nivaĉle) o 3.5. y 3.6. (ambas lenguas): el maká puede también valerse de las construcciones 3.2.b y 3.2.c.

(37a) Nivaĉle (SBP, Hechos 13: 33)

a'lhech avaatsha c'ôs'ash

$a$-tet $\quad a$-vátfa $\quad\left[k^{2}-p s^{2} a-\int_{\text {PRED }}\right.$

2-PRON-ENF 2-PRON 1POS(2s)-hijo-2-INST

'Eres tú mismo, eres tú, tú eres mi hijo'

(37b) Nivaĉle (SBP, Isaías 44: 17)

a'lhech c'adios'ash

a-tetf $\quad k^{2} a$-dios-'a- ${ }^{2}-5$

2-PRON.ENF 1POS(2s)-dios-2-INST

'Tú eres mi Dios' (cf. 50b)

(37c) Nivaĉle (SBP, Génesis 29: 15)

tanca acumyam'in

tanka a-kum-ya-m-'in

atesha lhama c'avelhtaj

NEG 2S.IRR-trabajar-1-BEN-INT de.balde aunque 1POS(2s)-prójimo-CON

'No vayas a servirme de balde, pese a que eres mi prójimo'

(37d) Nivaĉle (SBP, Salmo 16: 2)

avaatsha c'anvacle

a-vảt k ka-nvakle

2-PRON 1POS(2s)-señor

'Eres mi Señor'

El índice sufijado de (37e) y (37f) es de tercera persona. Debe tratarse del mismo fenómeno que en (18a), donde la tercera persona instrumental indica un tipo de relación algo excepcional (cambio de status o interrelación muy particular) o simplemente representa, de manera no obligatoria, el referente del nombre de interrelación personal. El verbo de relación interpersonal de (37e) está formado de la partícula coordinativa $t j i$ 'y, con' seguida por el morfema - $f a$ 'compañero'. En su empleo no marcado, $-t f i-f a$ es un nombre obligatoriamente poseído que significa 'compañero'. Aquí, el prefijo verbal doble se sustituye al prefijo posesivo.

(37e) Nivaĉle (SBP Oseas 2: 23)

a'lhechelh c'achifaseshelh

$a^{2}$-tetf-et $\quad k^{2} a-t f i-f a-s-e-f-e t$

2-PRON.ENF -PL 1POS(2s)-COn-COMP-3-INST-PL.COORD

'Ustedes son mi(s) gente(s)' 
(37f) Nivaĉle (SBP, Salmo 22: 10)

\begin{tabular}{|c|c|c|c|c|}
\hline $\begin{array}{l}\text { jôjjeclay } \\
\text { xpxeklay }\end{array}$ & $\begin{array}{l}\text { jôvji' } \\
x-p v-x i^{?}\end{array}$ & $\begin{array}{l}\text { lhcachi' } \\
\text { t-katfi? }\end{array}$ & $\begin{array}{l}\text { lhja } \\
t-x a\end{array}$ & $\begin{array}{l}\text { mimi, } \\
\varnothing \text {-mimi }\end{array}$ \\
\hline ya & 1s-estar-DENTRO & 3Pos-vientre & $\mathrm{F}-\mathrm{D}_{2}$ & 1POS-madre \\
\hline c'a & lios'esh & avaatsha & & \\
\hline$k^{2} a$ & dios-e-f & $a$-vảtfa & & \\
\hline $\mathrm{UB}_{1} 1 \mathrm{PC}$ & $S(2 s)$-dios-3-INST & 2-PRON & & \\
\hline
\end{tabular}

La frase verbal de (38a) incluye también una relativa ( $\sin$ relativizador) cuyo verbo modifica el predicado principal. Obsérvese la función distinta de los índices prefijados (en negritas). Tanto el nivaĉle como el maká carecen de formas verbales no personales, así que ambos miembros llevan el mismo prefijo. Obsérvese también el sufijo irrealis en el nombre de parentesco de (38b).

(38a) Nivaĉle (SBP, Marcos 1: 11)

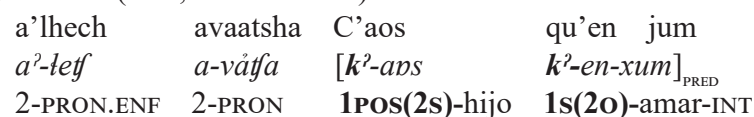

'Eres mi hijo [que] te quiero'

(38b) Nivaĉle (SBP, Génesis 12: 13)

\begin{tabular}{|c|c|c|c|c|}
\hline istaa & $\mathrm{ca}$ & at'esha & & c'ach'injô'a \\
\hline istả & $k a$ & $a-t^{2} e f-a$ & $k a$ & $k^{3} a-t$ 'inxp-a \\
\hline vale & $\mathrm{SUB}_{2}$ & 2S.IRR.decir-OBJ & $\mathrm{SUB}_{2}$ & 1POS(2s)-hermana.menor-IRR \\
\hline
\end{tabular}

(39) Nivaĉle (SBP, Salmo 22: 10)

$\begin{array}{lll}\text { ti } & \text { c'a Dios'esh } & \text { avaatsha } \\ t i & k^{2} a \text {-dios-e- } & a \text {-vảtfa } \\ \text { SUB }_{1} & \text { 1POS(2s)-dios-3-INST } & \text { 2-PRON }\end{array}$

'Eres mi Dios'

b) $[1 \operatorname{POS}(2 \mathrm{~s})-1 \mathrm{POS}-\mathrm{N}]_{\text {PRED. }}$ Esta configuración solo está documentada en maká, donde alterna con 3.2.c. El nivaĉle emplea otras construcciones, 3.2.a, 3.2.d, o 3.3.d. Ambas lenguas pueden expresarla con 3.5. o 3.6. Obsérvese que esta construcción combina el (doble) prefijo que indexa los dos participantes de un verbo monotransitivo con el índice nominal posesivo de primera persona. Obsérvese el doble plural ${ }^{16}$ del pronombre en (41a) y (41b) y otros ejemplos maká más abajo. Gerzenstein (1995: 175), la forma plural simple (pronombre plural -ekhewe) se opone al doble plural en la medida que este último se refiere a un maká y otra(s) personas que no forman parte de su grupo. Puesto que tanto la forma simple como la forma doble son muy comunes en el Nuevo Testamento, me parece más probable que este fenómeno esté ligado a la gran variedad (incluso cumulación dentro de

${ }^{16}$ En realidad, la marcación plural es cuádruple, sobre la base de una raíz pronominal -VkhV: -akha(masculino singular), -ekhe- (femenino singular o plural humano simple - lectura ambigua), -ekhe-we (plural humano), -ekhe-we-l (plural de grupo). 
una misma palabra) de morfemas plurales diversos en todas las lenguas chaqueñas de las familias mataguayo y guaykurú, o sea una un rasgo areal típico de todas estas lenguas. ${ }^{17}$ Para distinguir las diferentes modalidades de plurales que aparecen dentro en una misma palabra, empleo subíndices coindexados. Así, en la segunda palabra de (41a), $1 \operatorname{Pos}\left(2 \mathrm{~s}_{1}\right)$ y PL.COORD ${ }_{1}$, el subíndice ${ }_{1}$ marca la conexión entre un sujeto de segunda persona y el plural coordinativo $(2+3=$ ustedes $)$, mientras el lexema 'palabra' lleva el mismo subíndice que el plural que le corresponde $\left(\mathrm{PL}_{2}\right)$.

(40) Maká (WBT, Hechos 13: 33)

Akha' K'aya's

$a-k h a^{2} \quad\left[k^{2} a-y-a s\right]_{\text {PRED }}$

2-PRON 1POS(2s)-1POS-hijo

'(Sos vos,) sos mi hijo'

(41a) Maká (WBT, 2 Cor. 3: 2)

Ekheweli'ł k'eyilijeyi'ł

$\varnothing$-ekhewel-it $\quad k^{2} e$-yi-lixe-y-it

2-PRON.PL-PL.COORD 1 1 POS $\left(2 \mathrm{~s}_{1}\right)$-1 POS-palabra 2 -PL 2 -PL.COORD 1

'Ustedes son una carta [mi carta $=$ la que Cristo me confió para tú y él]'

(41b) Maká (WBT, Romanos 9: 26)

ekheweli'ł nite' k'eyitset'ił

Ø-ekhewel-it nite? $k^{2} e$-y-itset-it

2-PRON.PL-PL.COORD NEG 1POS(2s)-pueblo/aldea-PL.COORD

'Ustedes no son mi pueblo (lit. tú y él/ellos, no eres mi pueblo con él/ellos)'

(42) Maká (WBT, 1 Cor 4 : 14)

k'eyilitsi' tha

$k^{2} e-y i-$-its-it-ha

1POS(2s)-1POS-hijos-PL.COORD-INT

'Ustedes son mis hijos' (-lits es una forma plural irregular)

(43a) Maká (WBT, Juan 15: 14)

Ekheweli'ł k'eyijuwaikali'ł

$\varnothing$-ekhewel-it $\quad k^{2} e$-yi-xuwayka-l-it

2-PRON.PL-PL.COORD 1 1 POS $\left(2 \mathrm{~S}_{1}\right)$-1 POS-amigo $_{2}$-PL 2 -PL.COORD 1

'Ustedes son mis amigos' (cf. 58b)

(43b) Maká (WBT, Juan 8: 31)

yijaayi'ija

yixaayi-ixa

qu' k'eyijatshenheiyi'ilij

verdaderamente-ENF $\quad$ SUB $_{2} \quad 1$ POS $\left(2 \mathrm{~s}_{1}\right)$-1 POS-enseñar-NMLZ 2 -PL 2 -PL.COORD ${ }_{1}$-3-INST

'Ustedes serán realmente como discípulos míos'

${ }^{17}$ Para las lenguas de la familia mataguayo, se remite a los trabajos mencionados en la bibliografía. Carpio (2012b) y González $(2013,2016)$ tratan del tema a partir de la lengua toba (familia guaykurú). 
c) $[2 \mathrm{~s}-1 \mathrm{O}-\mathrm{N}]_{\mathrm{PRED}}$. Gerzenstein $(1995 \text { y } 1999)^{18}$ señala dos ejemplos maká aislados de su contexto, que incluyo aquí tentativamente (44 y nota 14 ). No he podido encontrar ningún ejemplo de esta configuración en el Nuevo Testamento. Tampoco se explica el inesperado irrealis del primer prefijo (realis: te-). Nótese en primer lugar que, en situación diádica (dialógica), tanto el nivaĉle como el maká pueden emplear formas de primera persona inclusiva $(1+2[+3])$ para referirse a relaciones recíprocas (somos amigos/vecinos). Para describir relaciones conversas (sos mi padre, sos mi hijo, sos mi esposa) siempre se da la posibilidad de presentar la relación desde la perspectiva del otro participante (soy tu hijo, soy tu padre, soy tu esposo). Ello vale también para las relaciones entre hermanos (hermano/a mayor vs. hermano/a menor). Faltan los términos generales hermano/a, si bien la Biblia hace gran uso de los términos ambiguos -vet, -tat-veteyetf (nivaĉle) y wit-xefe-yek (maká) 'pariente; familiar; prójimo', que muchas veces corresponden a la hermandad simbólica entre cristianos'. De acuerdo con Wicke \& Chase-Sardi (1969), -vet significa 'hermanastro de cualquier pariente consanguíneo de la generación de Ego', lo que me parece demasiado restringido puesto que también es muy frecuente en relatos tradicionales, donde se trata de cualquier 'compadre'. En lo que hace al maká, Gerzenstein (1999) la definición que propone para -xefe (no documenta -xefe-yek) es 'paisano; indígena; hermano (genérico); pariente', lo que corrobora la extensión bastante lata del término. El maká emplea también la estructura 3.2.b. Ambas lenguas pueden expresar esta misma relación con los esquemas 3.5. y 3.6.

(44) Maká (Gerzenstein 1994: 163)

$e-t s-k^{\prime} i n i x$

2s-10.IRR-hermano.menor

'Eres mi hermano menor'

Para expresar esta configuración, tanto el nivaĉle (3.2.d y 3.3.c - con relación inversa) como el maká suelen valerse de construcciones distintas.

d) $[\mathrm{PRON}]_{\mathrm{PRED}}+[\mathrm{D}+\mathrm{N}]_{\mathrm{REF}}$ Solo registrado en nivaĉle, en (45) con reduplicación opcional - del primer pronombre para énfasis. Alternativamente, el nivaĉle puede emplear la construcción 3.2.a., y ambas lenguas 3.5. o 3.6.

(45) Nivaĉle (SBP, Hebreos 1: 5)

a'lhech avaatsha na yaôs

$\left[a \text {-'tet }^{2} \quad a \text {-vảt } a\right]_{\mathrm{PRED}}\left[\begin{array}{ll}n a & y-a b s\end{array}\right]_{\mathrm{REF}}$

2-PRON.ENF 2-PRON D.M 1 POS-hijo

'(Eres tú mismo,) eres tú, mi hijo (aquí presente)'

3.3. $[\text { POS-N-PERS-INST }]_{\mathrm{PRED}}$. Esta configuración general está atestiguadas en ambas lenguas (con excepción de 3.3.c que solo es posible en nivaĉle). Aparece en siete combinaciones. En la construcción no marcada, el índice personal sufijado al predicado

${ }^{18}$ Además de reproducir el único ejemplo de la gramática (con la variante e-s-k'inix), el diccionario (Gerzenstein 1999) documenta también e-ts-iwket 'eres mi abuela'. 
de relación personal seguido por el aplicativo instrumental representa el sujeto. Tres estructuras marcadas (18c), (18e) y (18f) han sido analizadas arriba.

a) [3POS-N-1.PERS-INST $]_{\text {PRED }}$. Alternativamente, ambas lenguas pueden usar los tipos 3.4., 3.5. о 3.6 .

(46) Maká (WBT, Hebreos 1: 5, en negritas)

\begin{tabular}{|c|c|c|}
\hline $\begin{array}{l}\text { Yakha' } \\
y \text {-akha? }\end{array}$ & $\begin{array}{l}\mathrm{qu}, \\
q u^{?}\end{array}$ & $\begin{array}{l}\text { latataye'yij, } \\
\text { ta-tata-ye-yi-x}\end{array}$ \\
\hline 1-PRON & $\mathrm{SUB}_{2}$ & 3POS-padre-IRR-1-INST \\
\hline qa lakha' & qa' & ya'si'ij \\
\hline qa $\quad$-akha? & $q a^{?}$ & $y-a s-i-x$ \\
\hline 1-PRON & para.que & 1POS-hijo-3-INST \\
\hline
\end{tabular}

(47) Nivaĉle

lhcleslhyish

t-kles-et-yi-f

3POS-hijos-PL.COORD-1-INST

'Somos hijos de él/ella'

b) [3POS-N-2.PERS-INST $]_{\mathrm{PRED}}$. Alternativamente, ambas lenguas pueden emplear los tipos 3.4., 3.5. o 3.6.

(48a) Nivaĉle

lhcleselh'ash

t-kles-et-'a-f

3POS-hijos-PL.COORD-2-INST

'(Ustedes) son hijos de ella/él'

(48b) Maká (WBT, Gálatas 4:7 )

qa in hats las'ej

$q a$ in hats $t$-as-e- $x$

y SUB $_{2}$ ya 3POs-hijo-2-INST

'Así que eres su hijo'

(48c) aká (WBT, Romanos 6: 20)

\begin{tabular}{|c|c|c|}
\hline in mexe & tetinheyi'ł ej & pa' \\
\hline mexe & te-tin-he-y-it-e-x & $p-a^{?}$ \\
\hline $\mathrm{SUB}_{2}$ cuando & $\begin{array}{l}\text { 3POS-salvar/vivir.de-NMLZ-PL-PLCOORD-2 - }{ }_{1} \text {-INST } \\
\text { des estaban esclavos del pecado' (cf. } 58 \mathrm{~b} \text { ) }\end{array}$ & $\mathrm{D}_{\mathrm{v} 1}-\mathrm{M}$ \\
\hline
\end{tabular}

El ejemplo (48d) es una base verbal transitiva que expresa la misma relación que una predicación nominal. Obsérvese que se trata de un verbo básicamente transitivo, cuyo prefijo encierra los dos participantes, pero mantiene su forma verbal $\mathrm{S}+\mathrm{O}(48 \mathrm{c})$. $\mathrm{Si}$ bien no se puede considerar como una predicación posesiva nominal, contiene todos los ingredientes necesarios como para poder funcionar como tal. La estrategia de emplear un 
verbo en lugar del nombre permite evitar el cúmulo agramatical de prefijo predicativo y prefijo predicativo en una misma base. ${ }^{19}$

(48d) Maká (WBT, Juan 9: 28)

Akha' lijayan hakha' jukhew

$\varnothing$-akha? ti-xayan hakha? xukhew

2-PRON 2s(3o)-seguir D.M $\mathrm{IV}_{\mathrm{IV}} \mathrm{DEM}$ hombre

'Eres discípulo de aquel hombre (lit. tú sigues a aquel hombre)'

c) [2POS-N-1.PERS-INST $]_{\text {RRED }}$. No he podido documentar esta construcción en maká, que emplea las construcciones 3.4., 3.5. y 3.6., válida para ambas lenguas.

(49a) Nivaĉle

$a^{2}$-tata-et-yi- $\int$

2POS-padre-PL.COORD-1-INST

'Soy padre de ustedes' (49b) Nivaĉle

a-ka-tfen-atf-yi-f

2POS-MED.POS-enviar-NMLZ-1-INST

'Soy tu enviado'

(49c) Nivaĉle (SBP, 1 Reyes 18: 36)

ya'lhech yivaatsha acavoyish

ya'-tet $\quad y i$-vátfa a-kavo-yi-f

1-PRON.ENF 1-PRON 2POS-siervo-1-INST

'Soy yo, yo soy tu siervo'

d) [1 POS-N-2.PERS-INST $]_{\mathrm{PRED}}$. Para esta configuración, el maká emplea el tipo 3.2.c, o (3.4., ej. $58 \mathrm{~b}$ ), con dos predicados. Alternativamente, el nivaĉle puede valerse de las construcciones 3.2.a o 3.2.d. Ambas lenguas pueden también usar las estructuras 3.5. y 3.6.

(50a) Nivaĉle (SBP, 2 Sam 22: 29)

a'lhech yiqu'isjuyash'ash

$a^{2}-t e t y \quad y i-k^{2} i s x u y a f-{ }^{2} a-\int$

2-PRON.ENF 1POS-luz-2-INST

'Tú eres mi luz'

(50b) Nivaĉle (SBP, Salmo 140: 6)

\begin{tabular}{|c|c|}
\hline avaatsha & yi'Dios'ash \\
\hline$a$-vảtfa & $y i-\operatorname{dios}-^{-} a-\int$ \\
\hline $\begin{array}{l}\text { 2-PRON } \\
\text { 'Tú eres mi Dios' }\end{array}$ & $\begin{array}{l}\text { 1POS-dios-2-INST } \\
\text { (cf. } 37 \mathrm{~b} \text { ) }\end{array}$ \\
\hline
\end{tabular}

e) [1 POS-N-3.PERS-INST $]_{\text {PRED }}$. Atestado solo en maká (segundo nombre del ejemplo destacado con negritas), este tipo alterna con las construcciones 3.1.a, 3.3.d, 3.5. y 3.6, posibles en ambas lenguas.

${ }^{19}$ Para poder ser empleado con prefijos predicativos, tales nombres suelen intercalar entre el prefijo posesivo y la base la marca de poseedor desconocido. Cf. Gerzenstein (1999: 220): ye-wit'-ixatshen-ek 'soy alumno' y último ejemplo de (17), sacado de la misma obra. Sin embargo, la configuración de segunda persona puede expresarse con el prefijo verbal $k^{\prime} e-y$ - [1s(2o)-1pos-]. Para la diferencia entre esta glosa y la del ejemplo (43b), v. 3.2. y Tabla 6. 
$(50 \mathrm{c}=46)$ Maká (WBT, Hebreos 1: 5)

\begin{tabular}{llll} 
Yakha' & qu' & \multicolumn{2}{l}{ latataye'yij, } \\
$y$-akha? & $q u^{?}$ & \multicolumn{2}{l}{ ta-tata-ye-yi-x } \\
1-PRON & SUB $_{2}$, & 3POS-padre-IRR-1-INST \\
qa & lakha' & qa' & ya'si'ij \\
$q a$ & -akha? & $q a^{2}$ & $\boldsymbol{y}$-as-i-x \\
y & 1 -PRON & para.que & 1POS-hijo-3-INST
\end{tabular}

'Soy yo quien (seré) padre de él y él será mi hijo'

f) [2POS-N-3.PERS-INST $]_{\mathrm{PRED}}$. Esta construcción es exclusiva del nivaĉle. Para expresar esta configuración, el maká se vale del esquema 3.1.b. o 3.3.b. Ambas lenguas pueden expresarla mediante las estructuras 3.1.b, 3.5. o 3.6. El ejemplo maká (52b) no corresponde a ningún tipo de predicación nominal posesiva con nombre de relación interpersonal. Más bien, se trata de una forma presentativa, con sujeto no humano. El demostrativo/presentativo forma el predicado y el complejo determinante + poseedor + poseído forma un grupo referencial (52b).

(51) Nivaĉle

ac'utsfacheyesh

$a-k^{3} u t s-f a-t f e-y-e-\int$

2POS-apacentar-CMP-F-PL-3-INST

'Son tus compañeras' (52a) Nivaĉle

acavoesh

a-kavo-e- $\int$

2POS-gente-3-INST

'Es tu servidor'

(52b) Maká (WBT, Lucas 19: 20)

$\begin{array}{llll}\text { Yaqa patun } & \text { enewe'en } & \text { he' } & \text { aq'astai } \\ \text { ya-qa-patun } & \text { enewe-'en }_{\text {PRED }} & {\left[h-e^{?}\right.} & \left.a-q^{2} \text { astai }\right]_{\mathrm{REF}} \\ \text { 1POS-CL.POS-patrón } & \mathrm{D}_{\mathrm{H}} \text {-DEM-ENF } & \text { DI }_{\mathrm{v}}-\mathrm{F} & \text { 2POS-plata } \\ \text { '(Mi) señor, aquí está tu plata' } & & \end{array}$

g) [3POS-N-1INCL.PERS-INST $]_{\text {PRED }}$. Alternativamente, ambas lenguas pueden emplear las estructuras 3.4., 3.5. о 3.6.

(53) Nivaĉle

lhclesjooj

$t$-kles- $x \grave{o}-x$

3POS-hijos-1 INCL-INST

'Somos hijos de él/ella'
(54) Nivaĉle

(casvaatsha) lhcleselhjooj

(kas-vảtfa) t-kles-et-xỏ-x

(1INCL-PRON) 3POS-hijos-PL.COORD -1 INCL-INST

'(Nosotros) somos hijos de ellos

En el segundo verbo de (55), el plural $-s$ está coindexado con el sujeto de primera persona inclusiva -xỏ (sujeto de la predicación). 
(55) Nivaĉle (SBP, 2 Cor. 5: 20)

\begin{tabular}{|c|c|c|c|}
\hline ti & shincayôjatlhatay & ja & Cristo \\
\hline$t i$ & fi-nkaypx-xat-ta-t-ai & $x a$ & Cristo \\
\hline $\mathrm{SUB}_{1}$ & $\begin{array}{l}\text { (3A)1INCL.O-servir-CAUS-3-RR-3DIST } \\
\text { lhcheniasiooi }\end{array}$ & $\mathrm{D}_{2} \cdot \mathrm{M}$ & Cristo \\
\hline${ }_{t i}$ & $\boldsymbol{t}-\boldsymbol{t} \boldsymbol{t} \boldsymbol{e n}-\boldsymbol{x a} \boldsymbol{a}-\boldsymbol{s}-\boldsymbol{x} \boldsymbol{x}-\boldsymbol{x}$ & & \\
\hline & 3POS-enviar-NMLZ-PL - $1 \mathrm{INCL}_{1}$-INST & & \\
\hline
\end{tabular}

'Somos embajadores de Cristo' (lit. que nos ha puesto para servirlo Cristo que somos sus mensajeros)

(56) Maká (WBT, 1 Juan 3: 2)

$\begin{array}{lllll}\text { Yejefets, } & \text { hane'ej hats telits'inij } & \text { pa' } & \text { Intata } \\ \text { y-exefe-ts, } & \text { hane'ex hats te-lits-in-ix } & p \text { - } a^{\text {? }} & \text { in-tata } \\ \text { 1 Pos-amigo-PL ahora } & \text { ya } & \text { 3POS-hijos-1INCL-INST } & \mathrm{D}_{\mathrm{vI}} \text {-M } & \text { 1 INCL-padre } \\ \text { 'Amigos míos, somos hijos de Nuestro Padre' } & & \end{array}$

(57) Maká (WBT, Gálatas 4: 31)

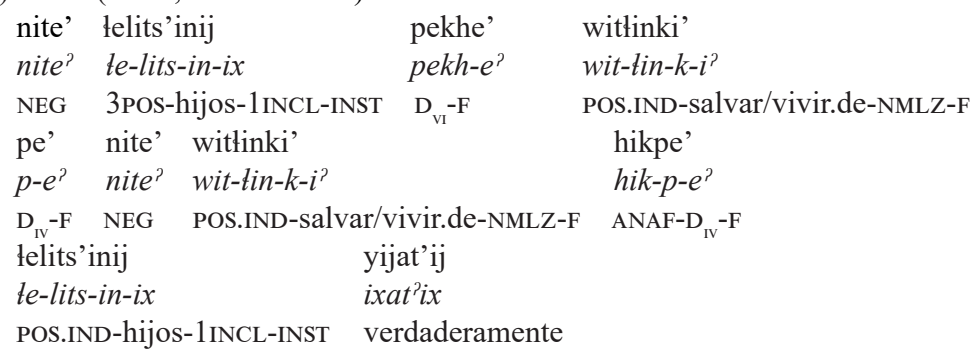

'No somos hijos de una esclava, de una no-esclava es de quien somos realmente hijos'

3.4. $[\mathrm{PRON} \sim \mathrm{CUANT}]_{\mathrm{PRED}}+\left[3 \mathrm{POS}-\mathrm{N}[\mathrm{D} \sim \mathrm{SUB}+\mathrm{N}]_{\mathrm{REF}}\right]_{\mathrm{PRED}}$. Se trata de una construcción bimembre formada con dos predicados. El primero consiste en un pronombre personal, un cuantificador (mayormente "(ser) todos" ${ }^{21} \mathrm{o}$ ambos. Los sujetos de ambos constituyentes son generalmente coindexados (58a), (58b) y (58c), pero no necesariamente (58d) y (59). En estas configuraciones, el poseedor debe ser de tercera persona y el sujeto de primera, primera inclusiva o segunda persona. No ha sido registrado con un sujeto de tercera persona, que emplea las estructuras 3.1.c., 3.5. y 3.6. para esta combinación. Este esquema está documentado en ambas lenguas. Alterna con 3.5. y 3.6.

El nombre - obligatoriamente poseído - 'hermanos (en Cristo)' se comporta de manera similar en maká (58a) y nivaĉle (58b). Sin embargo, puede recibir índices predicativos prefijados bajo una condición. En maká, el índice del poseedor debe ser indefinido (wit-, cf. nota 15). Esta opción no existe en nivaĉle, donde se emplea un índice 'postizo' (dummy)

${ }^{20}$ Después del índice de primera persona inclusiva, -ix es alomorfo del instrumental. En otros contextos, $-i-x$ es índice de tercera persona + instrumental.

${ }^{21}$ Los cuantificadores del maká y del nivaĉle tienen flexión verbal. 
de poseedor de tercera persona $(t-\sim t a-)$, que no desempeña otra función que la de permitir la flexión predicativa (desposesivo). ${ }^{22}$

(58a) Maká (WBT, Mateo 23: 8)

ekheweli'ł

[Ø-ekhewel-it

qa week

ewitjefeyeki'lipji'

2-PRON.PL-PL COORD y 3 s-ser.todos

$[e \text {-wit-xefe-yek-it-ipji }]_{\mathrm{PRED}}$

'Ustedes y todos son [quienes son] hermanos de ellos (nos amontonamos unos sobre otros)'

(58b) Nivaĉle (SBP, Mateo 23: 8)
alhacôm'aelh
ti alhtatvelheyechelh
$\left[a-t a k b m^{2} a-e t\right]_{\text {PRED }}$
ti $\quad[a \text {-t-tat-vet-eyet } f \text {-et }]_{\text {PRED }}$
2s-ser.todos-PL.COORD SUB 2 2S-DESP-RR-prójimo-PL.HUM-PL.COORD
'Todos ustedes son quienes son hermanos de ellos'

En (58c), el prefijo posesivo del nombre deverbal (segunda palabra) representa el sujeto de la predicación (el pronombre es redundante). Va seguido por una subordinada (para que hablemos en nombre de Cristo).

(58c) Maká (WBT, 2 Cor 5: 20)

\begin{tabular}{|c|c|c|}
\hline inekhewel & je'wit'ukinheyij & qu' ji'niyetij \\
\hline in-ekhewel & xe-wit'-ukin-he-y-i-x & $q u^{?} \quad$ xin-iyet $-i-x$ \\
\hline 1INCL-PRON.PL & 1INCL.POS-enviar-NMLZ-PL-3-INST & SUB $_{2}$ 1INCL.S.IRR-hablar-3-INST \\
\hline ka' lii & ha' Cristo & \\
\hline$k-a^{?} \quad t-i i$ & Cristo & \\
\hline $\mathrm{D}_{\mathrm{vi}}-\mathrm{M} \quad 3$ Pos-nol & bre $\mathrm{D}_{\mathrm{IV}}-\mathrm{M}$ Cristo & \\
\hline
\end{tabular}

'Somos embajadores de Cristo (lit. somos mensajeros-con para que hablemos en nombre de Cristo]'

En (58d), el pronombre de primera persona inclusiva es el sujeto (somos nosotros). Va seguido por un segundo predicado nominal, cuyo prefijo posesivo marca el poseedor (es su casa). Obsérvese la falta de concordancia entre el pronombre y el referente del segundo constituyente ([es] la casa del Dios que no muere). El poseedor propiamente dicho aparece, sin ninguna marca de poseedor, en una relativa (el Dios [que] no muere). Pese a la falta de concordancia gramatical entre los dos miembros de la construcción, resulta clara, desde una perspectiva pragmática, la identidad de los referentes de una estructura que fácilmente podría parafrasearse como ecuativa.

${ }^{22}$ Con otras palabras, éste va reforzada por una -v (ta.v-) o una -n (ta.n-) (para más detalles, ver Fabre 2016: 120-121). Si bien el contexto es diferente, este uso de una tercera persona dummy podría ser emparentado con la presencia de un índice de tercera persona mencionada abajo en wichí/'weenhayek (4.2). 
(58d) Maká (WBT, 2 Cor 6: 16)

inekhewel letsi' pa' Dios nite' wa'm

$[\text { in-ekhewel }]_{\mathrm{PRED}}\left[\begin{array}{l}\text {-etsi } \\ \text { ? }\end{array}\right.$

1INCL-PRON.PL 3POS-casa $\mathrm{D}_{\mathrm{v1}}$-M Dios NEG 3s-morir

'Nosomos somos la casa del Dios que no muere' (lit. Somos nosotros su casa [d]e Dios [que] no muere)

El ejemplo (59) es similar, y de igual manera que en (58d) muestra que ambos mientras son, por lo menos desde una perspectiva pragmática, correferenciales. Obsérvese que este enunciado es sinónimo de (43a). Sin embargo, la omisión del pronombre en (59) ocasionaría un cambio semántico drástico en la interpretación (ellos son mis amigos).

(59) Maká (WBT, Lucas 12: 4)

ekheweli'ł

yijuwaikal

$[\text { Ø-ekhewel-it }]_{\mathrm{PRED}} \quad[\text { yi-xuwaika-l }]_{\mathrm{PRED}}$

2-PRON.PL-PL.COORD 1POS-amigo-PL

''Ustedes son mis amigos ([los que son] mis amigos son ustedes)' (cf. 43a, 50a, 50b)

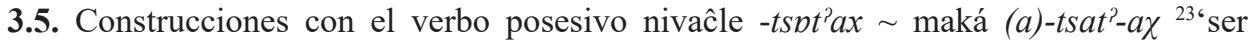
recto; ir derecho; tener; ser dueño de'. En ambas lenguas, los índices personales siguen el alineamiento inactivo (tercera conjugación). El índice sujeto, cuyo referente es el poseedor, tiene la misma forma que el objeto de un verbo transitivo, mientras el índice que corresponde a la cosa o persona poseída es sufijada y va seguida por el aplicativo instrumental (cf. hacerse con algo). Como el prefijo verbal corresponde al papel de ‘dueño' y el índice sufijado al de ‘poseído', la traducción puede ser, según el contexto, 'X (partiendo del prefijo) es dueño de Y' o 'Y (partiendo del sufijo) es súbdito de X'.

En (60), el nombre Dios (aquí en función predicativa) no permite la posesión directa (*ye-dios). La expresión de esta relación requiere del clasificador posesivo genérico -qe-. Como se puede apreciar, (60) consiste en dos predicados coordinados (él es mi dueño + es mi dios también).

(60) Maká (WBT, Juan 20: 28)

$\begin{array}{llll}\text { ¡Yatsat'axyij } & \text { qa } & \text { yeqe Dios } & \text { iye! } \\ \boldsymbol{y} \text {-atsat'ax-yi-x } & q a & \text { ye-qe-dios } & \text { iye } \\ \text { 3s-ser.dueño-1-INST } & \text { y } & \text { 1POs-CL.POS-dios } & \text { también } \\ \text { ‘Es mi señor y mi Dios también! (soy su súbdito)' }\end{array}$

En (61), el objeto (primera persona + aplicativo instrumental) del verbo -atsat'-ax corresponde a la entidad poseída. Se puede parafrasear como 'contar (en cuanto pertenencia) con algo/alguien'. El predicado lleva dos sufijos plurales, el primero coindexado con los súbditos (poseídos) y el segundo con el índice de segunda persona

${ }^{23}$ El maká tiene un sistema de tres fricativas posteriores sordas, velar, uvular y glotal. Nótese que, si bien tanto Gerzenstein como el Nuevo Testamento marcan la glotal $<\mathrm{h}>$, la $<\mathrm{j}>$ del NT corresponde a la $<\mathrm{x}>$ de Gerzenstein (AFI / x/), y la $<\mathrm{x}>$ del NT a la $<\mathrm{X}>$ de Gerzenstein (Asociación Fonética Internacional $/ \chi /$ ). 
(61) Maká (WBT, 1 Juan 4: 4)

ekheweli'ł

$\varnothing$-ekhewel-it

yatsat'etsi'1 ej

pa' Intata

2-PRON.PL.PL.COORD 3s-ser.dueño-PL-PL.COORD -2 -INST $\quad \mathrm{D}_{\mathrm{v}}-\mathrm{M} \quad$ IINCL.POS-padre

'Ustedes son [él es dueño de ustedes o ustedes son súbditos] de Nuestro Padre'

El ejemplo (62) sigue el mismo esquema: -ets (cognado con nivaĉle -(V)s, que no tengo registrado en este contexto $)^{24}$ marca el plural de la primera persona inclusiva -in. Recuérdese también (nota 20) que, después del sufijo de primera persona inclusiva y/o de la marca reflexiva-recíproca - $t$ - (62 y 63a), el alomorfo del instructivo es - $i x$. En otros contextos, $-i-x$ 'tercera persona' + 'instrumental'.

(62) Maká (WBT, 1 Juan $4: 6$ )

$\begin{array}{llll}\text { Inekhewel } & \text { yatsat'etsinij } & \text { pa' } & \text { Intata } \\ \text { in-ekhewel } & y \text {-atsat'-ets-in-ix } & p \text { - } a^{\text {? }} & \text { in-tata } \\ \text { 1 INCL-PRON.PL } & \text { 3s-ser.dueño-PL }-1 \text { INCL }_{1} \text {-INST } & \mathrm{D}_{\mathrm{V}} \text {-M } & \text { 1 INCL.POS-padre } \\ \text { 'Nosotros somos de Dios (lit. Nuestro padre es dueño de nosotros)' }\end{array}$

El sufijo reflexivo-recíproco en (63a) sirve para subrayar la interdependencia (y cercanía) de los participantes, al contrario de lo que ocurre en (62). El sujeto de primera persona inclusiva abarca la relación entre el dueño y los creyentes son inseparablemente unidos en un lazo de vida y muerte. La versión nivaĉle de este mismo versículo (64) no emplea el sufijo reflexivo-recíproco, y el prefijo de primera persona inclusiva solo expresa esta misma relación.

(63a) Maká (WBT, Romanos $14: 8$ )

nite' inekhewel qu' jinatsat'etsłe intij

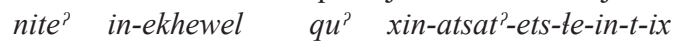

NEG 1INCL-PRON.PL SUB ${ }_{2}$ 1INCL.S.IRR-ser.dueño-PL $-\mathrm{RR}_{2}-1 \mathrm{INCL}_{1}-\mathrm{RR}_{2}$-INST

'[...que seamos o] no seamos de él mismo [el Señor]'

(63b) reduplica el concepto de predicación nominal posesiva con el verbo -atsat'-ax seguido por el nombre deverbal te-tin-he-i 'sus esclavos' (lit. sus de quienes ustedes viven).

(63b) Maká (WBT, Gálatas 4: 9)

Ye' me lisu'uni'ł qu' natsat'etsi'ł ej

$y e^{\text {? }}$ me ti-su'un-it $q u^{\text {? }} \quad n$-atsat'-ets-it-e-x

sí PREG 2 $2 \mathrm{~S}_{1}(30)$-querer-PL $\mathrm{SUB}_{2} \quad 3$ S.IRR-ser.dueño-PL-PL -2 -INST

qa' telinheyi'ił ej iye

$q a^{\prime} \quad$ te-tin-he-y-it-e-x$\quad$ iye

para.que 3POS-salvar/vivir.de-NMLZ $-\mathrm{PL}_{1}-\mathrm{PL}_{\mathrm{CORD}}-\mathrm{CO}_{2}$-INST también/otra vez

¿o quieren ustedes ser de ellos y ser de nuevo sus esclavos?

${ }^{24}$ La traducción nivaĉle literal de este versículo dice 'nosotros que venimos/somos descendientes de Dios' $<$ casvaatsha ti shtantô'lha pa Dios> -/kas-vátfa ti Jtan-tp't-a pa Dios/. La forma plural, -tswt'a-s, solo se emplea en la acepción 'ser recto/derecho'. 
(64) Nivaĉle (SBP, Romanos 14: 8)

shtantsot'ajshelh

ftan-tspt'ax-Ø-f-et

pa Lhcaanvacle

pa t-kaa-nvakle

1INCL.S-ser.dueño-3-INST-PL.COORD D.M 4 3POS-MED.POS-señor

'Somos del Señor (lit. Es dueño de nosotros [muchos], el Señor [de otros señores])'

(65) ejemplifica una forma negativa con el mismo verbo.

(65) Nivaĉle (SBP, Juan 4: 18)
jôque ajayataj
shta ninatsôt'ajesh
$[x p-k e \quad \text { a-xaya-tax }]_{\mathrm{REF}} \quad$ fta
$[\text { ni-na-tspt'ax-e- }]_{\mathrm{PRED}}$

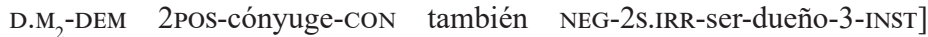
'Éste tu (supuesto) marido no es tuyo'

3.6. Construcciones con el verbo 'venir; provenir; ser causa de' (nivaĉle -tp̉t maká $\left.-t a^{2} t\right)$ para indicar relaciones de descendencia bilateral directa (padres/abuelos/abuelas/ ancestros). Las terminologías de parentesco del área chaqueña han sido estudiadas en detalle por Braunstein (1983). Para el nivaĉle, se dispone también del estudio de Wicke \& Chase-Sardi (1969a/b). Lamentablemente, ninguna de estas fuentes menciona el término formado sobre el verbo venir, aunque está bien representado en textos recopilados entre los nivaĉle. ${ }^{25}$ Moser \& Marlett (1989) han registrado varios nombres de parentesco seri (lengua aislada de Sonora, México) derivados de verbos, entre los cuales destaca precisamente 'venir' para referirse a los padres. El verbo 'venir' puede emplearse en construcciones sintéticas $(66,57 \mathrm{~d}, 67 \mathrm{e})$. No estoy seguro que los empleos de 'venir', en (67b), (67c), y (67e) puedan ser considerados como ejemplos de estructuras de predicación nominal posesiva con nombres de relación sin la presencia de la frase determinativa que le sigue. Así, podrían mejor analizarse como ejemplos de estructuras analíticas bimembres.

(66) Maká (WBT, Mateo 23: 31)

\begin{tabular}{|c|c|c|}
\hline ekheweli'ł & latata'tiłets & kekhewe' \\
\hline Ø-ekhewel-it & ta-ta't-it-Ø-ets & kekhe-we? \\
\hline $\begin{array}{l}\text { 2-PRON.PL-PL.COORD } \\
\text { nilanju'kii }\end{array}$ & $\begin{array}{l}2 \mathrm{~s}_{\mathrm{I}} \text {-venir-PL.COORD } \\
\mathrm{ke}^{\prime} \text {-3-OBJ/LOC }\end{array}$ & $\mathrm{D}_{\mathrm{v}}-\mathrm{PL}$ \\
\hline$n i-l a n-x u^{2}-k i i$ & $k-e^{?} \quad$ profeta-iki & \\
\hline 3s(3o)-matar-PL.OBJ-PL.SUJ & $\mathrm{D}_{\mathrm{v}}-\mathrm{PL}$ profeta-ENF & \\
\hline
\end{tabular}

La versión nivaĉle del ejemplo anterior (67a) emplea la estructura analizada arriba en 3.3 .

${ }_{25}$ Además de los textos recopilados bajo la supervisión de Seelwische, se pueden mencionar las narraciones de vida monolingües publicadas en Tierra Libre (2014), si bien no contiene ejemplos de predicación con nombres de relaciones interpersonales. Hay también numerosos ejemplos de esta palabra (también en su forma predicativa) a lo largo y ancho de la Biblia. 


\section{FABRE - LA PREDICACIÓN NOMINAL POSESIVA...}

(67a) Nivaĉle (SBP, Mateo 23: 31)

\begin{tabular}{|c|c|c|c|c|}
\hline yijô' & ti & lhcleselh'ash & yiclônsha'ne & lhapesh \\
\hline yixp? & $t i$ & t-kles-et-ia-f & $y i-k l p n-\int a^{2} n e$ & tapes \\
\hline seguro & $\mathrm{SuB}_{1}$ & 3POS-hijos-PL.COORD - -2 $_{1}$-INST & $\mathrm{D}_{4}$-PL.HUM $3 \mathrm{~s}(3 \mathrm{O})$-matar-PL.OBJ & PASADO \\
\hline capi & chiytôjata & pava & ti vaclan & \\
\hline$k a-p i$ & $t i-y-t p y-x a t-a$ & $p a-v a$ & $\varnothing$-vaklan & \\
\hline${ }_{3}$-PL.HUM & S.IND-3s $(30)-s a b$ & ber-CAUS-META $\quad \mathrm{D}_{4}$-PL.NO.HUM & I $\mathrm{SUB}_{1}$ 3s-acontecer & \\
\hline
\end{tabular}

(67b) Nivaĉle (SBP, Romanos 11:1)

$\begin{array}{llllll}\text { Jatô'lha } & \text { shta } & \text { ja } & \text { tc'asham } & \text { pa } & \text { Benjamín } \\ x a-t t^{\prime}-a & \int t a & x a & \varnothing \text {-tk'a-fam } & \text { pa } & \text { Benjamín } \\ \text { 1s-venir-PROx } & \text { también } & \text { D.M.M } & \text { 3s-reunirse-PERL } & \text { DM } & \text { Benjamín }\end{array}$

'Yo también soy descendiente de la tribu (lit. de entre los reunidos) de Benjamín'

(67c) Nivaĉle (SBP, 2 Corintios 11: 22)

\begin{tabular}{|c|c|c|c|c|c|}
\hline ¿Tôlhshaam & japi & Israel & lhcles? & Ya chi' & shta \\
\hline$\varnothing-t b t-\int a ̉ m$ & $x a-p i$ & Israel & t-kles & $y a-t i^{?}$ & fta \\
\hline 3S-venir-PERL & $\mathrm{D}_{2}$-PL.HUM & Israel & 3POs-hijos & $1 \mathrm{~s}-(\mathrm{ser}) \mathrm{COORD}$ & también \\
\hline jatôôlhsham & japi & Israel & lhcles & & \\
\hline$x a-t p t-\int a m$ & $x a-p i$ & Israel & t-kles & & \\
\hline 1s-venir-PERL & $\mathrm{D}_{2}$-PL.HUM & Israel & 3POs-hijos & & \\
\hline
\end{tabular}

‘¿(Ellos) son descendientes de los israelitas? Y yo también soy descendiente de los israelitas'

(67d) Nivaĉle (SBP, 2 Timoteo 2: 8)

\begin{tabular}{|c|c|c|c|c|}
\hline Jô lhech & tôlheyshi & pa & lhcaanvacle & David \\
\hline$x p-t e t$ & $\varnothing-t p t-e-i-\int i$ & $p a$ & t-kaa-nvakle & David \\
\hline $\begin{array}{l}\mathrm{D}_{2}-\mathrm{ANAF} \\
\text { 'El (es qu }\end{array}$ & 3s-venir-3-DIST-INH & D. $\mathrm{M}_{4}$ & 3POS-MED.POS-señor & David \\
\hline
\end{tabular}

(67e) Maká (SBP, 2 Timoteo 2: 8)

\begin{tabular}{|c|c|c|c|c|c|}
\hline hik & hakha' & iye & ta'łets & pa' & David'ik'i \\
\hline hik & hakha? & iye & $\varnothing$-ta't-ets & $p-a^{?}$ & David-ik'i \\
\hline ANAF & 3PRON & también & 3s-venir-OBJ/LOC & $\mathrm{D}_{\mathrm{vI}}-\mathrm{M}$ & David-ENF \\
\hline
\end{tabular}

En su forma más simple, ha-tat-ets, que aparece en (68), significa 'vengo de'. Al tratarse de parentesco humano, indica descendencia directa y no colateral (excluye hermanos/hermanas, y tíos/tías), probablemente bilateral, como en nivaĉle. El sufijo -(w) ets es un aplicativo locativo que marca el punto de partida o la meta de la actividad, de acuerdo con la semántica del verbo. En otros contextos, -(w)ets puede marcar el objeto. El índice de segunda persona entre el plural coordinativo y el locativo se refiere a Dios, como la entidad a la cual se dirige el poeta ("como dijeron algunos poetas de ustedes". En un cotejo de varias versiones castellanas e inglesas de este versículo, ninguna se aparta de la tercera persona con referencia a Dios. Por esta razón, el maká emplea el plural coordinativo (yo y él/ellos - plural exclusivo) La versión sigue el esquema general, lo que 
explica el plural inclusivo (69). El locativo proximal de (69) tiene la misma función que $-(w)$ ets en (68).

(68) Maká (WBT, Hechos 17: 28)

$\begin{array}{lllll}\text { yekheweli'ł } & \text { iye } & \text { hata'ti'l ewets } & \text { iye } & \text { Dios } \\ y \text {-ekhewel-it } & \text { iye } & {[\text { ha-ta't-it-e-wets }]_{\text {PRED }}} & \text { iye } & \text { Dios } \\ \text { 1-PRON.PL-PL.COORD } & \text { también } & \text { 1s-venir-PL.COORD-2-OBJ/LOC } & \text { también } & \text { Dios }\end{array}$

'Nosotros también somos linaje de Dios (lit. vengo con él/ellos [exclusivo] de ti, Dios)

(69) Nivaĉle (SBP, Hechos 17: 28)

casvaatsha shtatô'lhelhavatam pa Dios

kas-vảtfa fta-tot-et-a-vatam pa Dios

1INCL-PRON 1INCL.S-venir-PL.COORD-PROX-PL.GRUPO D.M ${ }_{4}$ Dios

'Nosotros, todos descendemos de Dios'

\subsection{Fenómenos emparentados}

La literatura lingüística hace referencia a otras construcciones emparentadas con la predicación nominal con nombres de relaciones interpersonales. Según las lenguas, pueden formar frases nominales y/o verbales. Evans (2000) describe construcciones con verbos de parentesco y otras relaciones interpersonales en varias lenguas indígenas australianas, norte- y mesoamericanas. No todos pueden emplearse igualmente en función predicativa o referencial. Además, hace una diferencia básica entre predicados verbales ecuativos (canónicos), del tipo soy su hijo, y otros que conllevan un matiz adicional como tratar como/considerar o llamar a alguien con el nombre. Los verbos de parentesco, tanto en frases nominales como predicativas, no son desconocidos en zonas del Cono Sur, tal como puede apreciarse en trabajo de Evans, Golluscio \& Mellico (2010) sobre el mapudungun (70). Si bien todos los ejemplos aparecen en tercera persona, Smeets (2007) ilustra también una construcción similar, pero con morfología distinta (verbalizador y recíproco)- en primera persona dual (71).

(70) Mapudungun (Evans, Golluscio \& Mellico 2010: 64)

wenïy-wen wen̈̈y-wen-nge-i-ngu

amigo-DIAD amigo-DIAD-ESIVO-INDIC-3DUAL

'Amigos' 'Los dos son amigos'

(71) Mapudungun (Smeets 2007: 290 - con glosa ligeramente modificada por el autor)

peñi-ye-w-y-Ø-u

hermano-VBLZ-RR-INDIC-NO.SG-DUAL

'(Los dos) somos hermanos'

El maká y el nivaĉle emplean el sufijo 'compañero/a' (nivaĉle $-f a[\mathrm{~m}],-f a-t f e$ [f] maká - $f e^{\prime}[\mathrm{m}],-f e-k i$ ' [f] aparece a menudo una sílaba epentética entre la raíz y la marca de derivación) para derivar nombres de relaciones interpersonales (pero no de parentesco con la excepción de -t' $f^{\prime} a k f a$ 'cónyuge' - aparentemente lexicalizado), que pueden funcionar como frases nominales o predicativas. 
(72) Maká (Gerzenstein 1999: 303)

-qatxat 'olla' -qatxat-efe 'compañero de cocina', -qatxat-efe-ki' 'compañera de cocina' $k^{3} a-y$-aq-atxat-efe?

1s(2o)-1 Pos-olla-CMP

'Soy tu compañero de cocina'

(73) Maká (Gerzenstein 1999: 286)

-otoy 'bailar' -otoy-hife'

$k^{\prime} \mathrm{o}$-y-otoy-hife?

1s(2o)-1 POs-bailar-CMP

'Soy tu compañero de baile'

(74) Nivaĉle (SBP 1994, Jeremías 3: 4-5)

ac'utsfaeshyam ti yanich'ayiche

$a-k^{2} u t s-f a-e-f-y a-m \quad t i \quad y a-n i t f a y i t f e$

2s-apacentar-CMP-3-INST-1-BEN SUB $_{1}$ 1s-ser.muchacho

'Eres mi compañero de juventud (lit. que soy/era muchacho)'

(75) Nivaĉle

yicumjafaesh ja Juan

yi-kumxa-fa-e-f xa Juan

1 POs-trabajar-CMP-3-INST D.M 2 Juan

'Juan es mi compañero de trabajo'

(76) Nivaĉle (SBP 1994, Oseas 2: 2)

tanca lhch'acfaayish

tanka t-t's ak-fa-a-yi-f

NEG 3POS-?-CMP-IRR-1INST

'No soy el marido de ella' (?-CMP = cónyuge [compañero de/en ?] $)^{26}$

Otras construcciones con verbos de relaciones interpersonales se forman con un subtipo de verbos que normalmente funcionan como lexemas que denotan pares con defectos y cualidades corporales. En maká y en nivaĉle, el miembro negativo contiene el verbalizador -met (maká) o -mat (nivaĉle). La forma positiva es, respectivamente,

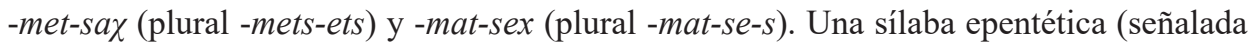
con un punto) puede aparecer ante el sufijo. (77a) y (77b) ilustran casos canónicos, que indican defectos y cualidades de un solo participante. Probablemente, esta formación es productiva, por lo menos en nivaĉle.

(77a) Nivaĉle (Seelwische 1990 y notas de campo del autor) $-f o^{\text {? 'pie' } \rightarrow-f o^{\text {? }}-\text { mat }_{1} \text { 'tener defectos en los pies' } \rightarrow-f o^{\text {'}} \text {-mat-sex }}$ 'tener los pies sanos' -kafof 'pulmón' $\rightarrow$-kafif-i.mat ' 'tener enfermedad pulmonaria'

-px 'piel' $\rightarrow$-px-e.mat ${ }_{1}$ 'tener enfermedad de la piel', -px-e.mat-sex ' 'tener buena piel; se curado de una enfermedad de la piel'

-tps-ex 'ojo', -tw-'vat 'vista' $\rightarrow$-tp'-mat ${ }_{1}$ 'tener mala vista', -tp-mat-sex ${ }_{1}$ 'tener buena vista'

${ }^{26}$ En otro contexto, el nombre en posición predicativa podría significar 'no soy la esposa de él'. Solo cuando el nombre 'cónyuge' ocurre dentro de una frase determinativa, la presencia del determinante señala el género. 
(77b) Maká (Gerzenstein 1999; WBT 2013)

-'ax 'piel' $\rightarrow$-ax-a.met 0 'tener enfermedad de la piel'

-tawex 'vientre; interior (sede de emociones)' $\rightarrow$-itawxe-met ${ }_{0}$ 'estar preocupado'

$? \rightarrow$-iwelki-met 0 'ser cojo'

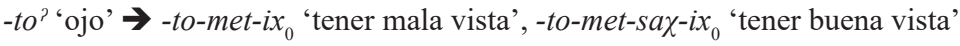

Un subtipo de estos verbos (77c) indica relaciones interpersonales o actividades sociales, muy escasamente términos de parentesco. En todos estos casos, ya no se trata de una particularidad corporal particular, como en (77a-b), sino de una relación negativa o positiva entre dos o más individuos, o cuando menos de importancia para el grupo social. No he podido encontrar ejemplos claros de este tipo en maká, sino quizás (77d).

(77c) Nivaĉle (Seelwische 1990 y notas de campo del autor)

- axe $^{\text {? 'presa' } \rightarrow \text {-axe'-mat }}{ }_{1}$ 'tener mala presa', axe'-mat-sex ${ }_{1}$ 'tener/volver con buena presa'

-ei-fa 'tocayo (nombre-compañero)' $\rightarrow$-ei-fa-mat ${ }_{1}$ 'tiene un tocayo malo', -eifa-mat-sex 'tiene un tocayo bueno'

$-k u f a-i_{2}$ 'tener/dar regalo' $\rightarrow \quad-k u f a n-x a . m a t-s e x_{1}$ 'ser generoso'

$-y x_{4} i_{4}$ 'ordenar' $\rightarrow-y$ pxi-xa.mat-sex ${ }_{1}$ 'saber ordenar; hacerse respetar'

$-t^{\prime} a n_{2}$ 'escuchar' $\rightarrow-t$ ' $\rightarrow$ an-xa.mat ${ }_{1}$ 'ser desobediente', -tf'an-xa.mat-sex ${ }_{1}$ 'ser obediente'

-vet 'pariente varón', vet-tfe 'pariente femenino' $\rightarrow$-vet-i.mat 'tener parientes malos',

-vet-i.mat-sex 'tener parientes buenos', -vet-tfe-mat 'tener mujeres malas como parientes'

(77d) Maká (SBP 1994)

-ika? 'marcar; dibujar' $\rightarrow$-ika-met-wet-xut 'golpearse unos a otros; darse piñas'

-ithayi $i_{i}$ 'trabajar' $\rightarrow$-ithayi-met ${ }_{0}$ 'trabajar con dificultad', -ithayi-met-sa $\chi_{0}$ 'trabajar con facilidad'

\section{Predicación nominal posesiva con nombres de relaciones interpersonales en wichí/'weenhayek y chorote}

Lo que sigue es un breve panorama de la predicación nominal en las otras dos lenguas de la familia mataguayo, el wichí (con su variante 'weenhayek) y el chorote. En lo que sigue, la grafía original de las fuentes, más o menos fonológica, ha sido mantenida.

\subsection{Chorote.}

De acuerdo con Carol (2014: 153), esta lengua ${ }^{27}$ cuenta con cuatro sufijos de predicación nominal, que corresponden a las primera y segunda personas, singular y plural (78a). Cada uno de estos sufijos combina un índice persona con el aplicativo locativodativo - $m$. Una serie de procesos morfofonológicos bastante complejos acarrea frecuentes problemas de segmentación en chorote. Es importante señalar que estos sufijos no solo

${ }^{27}$ Según el autor citado, el número de hablantes de las tres variedades del chorote no supera los 3.000. 
funcionan como índices predicativos, sino muchas veces como marcadores de objeto, con (78b) y (78c) o sin el aplicativo dativo/locativo.

(78a) Sufijos de predicación nominal en chorote (Carol 2014: 153)

1SG. -k'im 1PL. -ts'em

2sG. -'am 2PL. -'a-s-em

(78b) Chorote, Carol (2014: 265)

$i$-'yen-k'i'm

3A-mirar-1SG+LOC/DAT

'Me cuida'

(78c) Chorote, Carol (2014: 261)

$t$-an-k'i

3s-1lamar-1sG+LOC/DAT

'Me llama'

Ahora bien, estos sufijos de predicación nominal pueden agregarse directamente a nombres poseídos, de acuerdo con el esquema ÍNDICE DE POSEEDOR + NOMBRE + Índice personal de predicación. Carol observa que en (79a), los índices personales de predicación tienen alcance sobre la secuencia índice personal de poseedor + nombre. Obsérvese además que en (79a), el sufijo plural está coindexado con el poseedor. Tanto en (79a) como en (80), la forma plural puede considerarse como irregular o supletiva (el singular es -as). En (81b), la presencia del subordinador $t i$ (opcional en muchos casos) es un índice adicional de que la palabra que sigue es tratada como un verbo. En lo que sigue, se ilustran diversas combinaciones.

(79a) Chorote (Carol 2014: 153)

[ji-nya-yis]-k'im

3POS-padre-PL-1SG.LOC/DAT

'Soy (el) padre de ellos'

(79b) Chorote (SBA, Gálatas 4: 7)

jl-ajts-'a'm

3POS-hijo-2sG.LOC/DAT

'Eres su hijo'

(80) Chorote (SBA, 1 Juan 3:2)

ijiate Si-nia' [ji-lis]-ts'em

verdadero 1PL.POS-padre 3POs-hijos-1PL.LOC/DAT

'...de veras, somos hijos de Nuestro Padre'

(81a) Chorote (Carol 2014: 153, 363; SBA, Cor. 6: 18)

'a-s-a'am ja [i-lis-a]-'a-s-e'm

2-PL-PRON PROSP 1SG.POS-hijos-IRR-2PL.LOC/DAT

'...ustedes serán mis hijos'

(81b) Chorote (SBA, Hechos 13: 33)

$\varnothing$-a'am ti $y$-ajts-'a'm

2-PRON SUB 1 1POS-hijo-2sG.LOC/DAT

'Tú eres mi hijo' 
Los ejemplos arriba mencionados son muy parecidos a los tipos maká y nivaĉle tratados en $\S 3.3$, pero solo cuando el sujeto de la construcción es una primera o segunda persona (singular o plural). Cuando el sujeto es de tercera persona, se emplea la misma construcción que maká/nivaçle $\S 3.1$.

La tercera persona sujeto no lleva ninguna marca. Se construye como en nivaĉle y maká, de acuerdo con el principio $[\mathrm{D}+(\mathrm{POS}) \mathrm{N}]_{\mathrm{RFF}} \quad \rightarrow \quad[(\mathrm{POS}) \mathrm{N}]_{\mathrm{PRED}}{ } \quad$ Obsérvese además que en (82) la referencialidad del primer miembro de la construcción se debe a la anteposición de $\mathrm{D}$ a un verbo $([\mathrm{D}+\mathrm{V}]=$ frase referencial (cf. Tabla 1 , que vale también para el chorote). Además, se confunde con un tipo de relativa común en las lenguas mataguayo. Confróntese (82) con sur versiones maká (32) y nivaĉle (65), que ilustran tres construcciones diferentes.

(82) Chorote (SBA, Juan 4: 18)

s siunie $\left._{\text {jl-e-jiap }}\right]_{\mathrm{REF}} \quad\left[j e \quad a-k^{\prime} \text { ienjla }\right]_{\mathrm{PRED}}$

D.M 1 2s-estar-JUNTO NEG 2POS-cónyuge

'El [con quien] estás no es tu marido'

El ejemplo (83a) ilustra, con sujeto de tercera persona, el esquema $[\mathrm{D}+\mathrm{POS}-\mathrm{N}]_{\mathrm{RFF}} \rightarrow[\mathrm{POS}-\mathrm{N}]_{\mathrm{PRED}}$

(83a) Chorote (SBA, Juan 8: 39)

Abraham, pa-k si-nia'

Abrahám, $\mathrm{D}_{6}$-TOP 1PL.POS-padre

'Abrahám, él es nuestro padre'

(83b) y (83c) ilustran el verbo 'venir' en el sentido de 'ser descendiente de' (cf. 3.6).

(83b) Chorote (SBA, Hebreos 7: 5)

siupapo pa'yijis t'ipi pet ti tajla'm

siu-pa-po pa'yi-jis t'i-pi pet ti Ø-tat-am

DEM-D 6 -PL.HUM sacerdote-PL CONJ-PREM también SUB $_{1} 3$ s-venir-LOC/DAT

pak Leví

$\mathrm{D}_{6}$-TOP Levi

'Aquellos sacerdotes que son descendientes d[el ancestro] Leví)'

(83c) Chorote (SBA, 2 Timoteo 2: 8)

jla'am ti tajla'm papo David jilist'ipi

jl-a'am ti Ø-tajl-am pa-po David ji-lis-t'i-pi

3-PRON SUB 3s-venir-LOC/DAT D -PL.HUM David 3POS-hijos-CONJ-PREM

'(Jesús) es descendiente de los hijos de David'

\subsection{Wichí / 'Weenhayek}

Las estimaciones del número de hablantes pertenecientes al conjunto wichí/'weenhayek varian entre 40.000 y 60.000 (4.115 hablantes en Bolivia, y el resto en la Argentina). ${ }^{28}$ Se trata, pues, de la lengua mataguaya hablada por el mayor número

${ }^{28}$ V. Nercesian (2009) y Braunstein (1993). 
de personas. La cumulación de índices posesivos y predicativos en una misma base es también posible en wichí, por lo menos en dos variedades del noroeste (Lunt 1999 y Alvarsson \& Claesson 2014; Claesson 2016). Al contrario de lo que ocurre en las otras tres lenguas mataguayo, donde los enunciados referenciales (frases nominales, formadas a partir de un nombre o de un verbo) se distinguen por la presencia obligatoria de un determinante, las frases nominales del wichí/'weenhayek no requieren de la presencia de determinantes. Cuando éstos aparecen, siempre van sufijados al nombre. Así, cuando una frase wichí/'weenhayek Pos-N va combinada con un predicado, es una frase nominal (84-86, primer ejemplo de 87, 91-93), pero en otros contextos, puede ser predicativa (8890, 94-96). Construcciones ecuativas bipartitas PRED + POS- $\mathrm{N}_{\mathrm{RFF}}$ similares han sido ilustradas arriba en maká (21) y nivaĉle (22). Los ejemplos wichí y 'weenhayek se citan con las grafías originales, generalmente fonológicas, de las fuentes.

Claesson (2016) documenta dos construcciones 'weenhayek. La primera involucra dos bases, cada una con el prefijo personal que le corresponde ${ }^{29}$ (84-86). Esta estructura está formada a partir de la de predicación nominal no posesiva, donde cópula y nombre predicativo llevan la misma serie de índices personales (87).

(84) 'Weenhayek (Claesson 2016: 18)

['aa-hâpe $]_{\mathrm{PRED}}[\text { 'oo-wuk }]_{\mathrm{REF}}$

2s-COP 1POS-dueño

'Eres mi dueño'

(85) 'Weenhayek (Claesson 2016: 18)

['o-hâàpe $]_{\mathrm{PRED}}[\text { 'a-wuuk }]_{\mathrm{REF}}$

1S-COP 2POS-dueño

'Soy tu dueño'

(86) 'Weenhayek (Claesson 1981-1996, Juan 4:18 - segmentación y glosas AF)

$[\text { tok Ø-hààpe }]_{\mathrm{PRED}}[\text { ha-ky'eejwah }]_{\mathrm{REF}}$

NEG 3s-COP 2POS-cónyuge

'No es tu esposo'

(87) 'Weenhayek (Claesson 2016: 17)

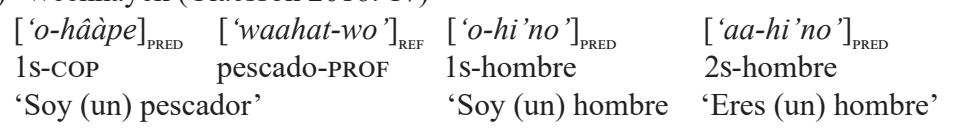

Otra construcción combina las dos marcas, prefijadas a una misma base (88-89). Se puede también emplear en la forma reflexiva (90). Cuando los dos índices van prefijados, opera el mismo alineamiento jerárquico $1>2>3$ que el que también rige los casos de prefijación de ambos índices sujeto y objeto en construcciones transitivas. ${ }^{30} \mathrm{El}$ índice más

\footnotetext{
${ }^{29}$ Hay cuatro series de índices pronominales en wichí/'weenhayek: 1) posesivos, 2) predicativos y cópula, 3) sujeto, y 4) objeto. La primera se usa con nombres obligatoriamente poseídos, la segunda, con nombres predicativos y cópula. Las tercera y cuarta se usa con verbos.

${ }^{30} \mathrm{El}$ mismo alineamiento jerárquico tiene un papel central en las construcciones transitivas de nivaĉle y del maká. Para una descripción del fenómeno (muy similar al del maká), ver Fabre (2012).
} 
alto va primero, cualquiera sea su función, ya como poseedor o sujeto de la predicación posesiva. En los dos primeros ejemplos de (88) y (89) y los dos ejemplos wichí en (96) el índice más alto aparece en la margen izquierda de la palabra En los dos ejemplos wichí en (95) el participante más alto se encuentra en la margen derecha de la palabra y el (96). Si solo uno de los índices va prefijado - y el segundo tampoco aparece como sufijo - solo se mantiene el índice más alto de la jerarquía de verbos transitivos. El mismo fenómeno se observa en construcciones transitivas con los índices sujeto y objeto. Obsérvese que el wichí/'weenhayej y el maká comparten la posibilidad de prefijar un índice personal verbal con un posesivo. Sin embargo, esta posibilidad es más restringida en maká, en que solo fue registrado $1 \mathrm{~A}(2 \mathrm{O})=1 \mathrm{POS}(2 \mathrm{~s})+1 \mathrm{POS}(40-43)$, mientras el wichí/'weenhayek permite combinar, siempre con posesivo de tercera persona, la primera, segunda y primera persona inclusiva de sujeto $(88,89,95,96)$. Esta construcción es muy parecida a la del nivaĉle tratada bajo § 3.2.a. Si bien los dos índices aparecen prefijados tanto en 'weenhayek como en wichí, su orden respectivo es regido por un alineamiento típicamente jerárquico $(1>2>3)$ que no considera la función del índice sino su posición en la jerarquía. Nótese que cuando un sujeto de tercera persona va combinado con un poseedor de primera o segunda, hay que emplear el esquema con cópula.

(88) 'Weenhayek (Claesson 2016: 18)

$\begin{array}{lll}{\left[{ }^{\prime} o-l a-w u u k\right]_{\text {PRED }}} & {[\text { 'aa-la-wuk }]_{\text {PRED }}} & {[l a-w u u k]_{\text {PRED }}} \\ 1 \text { s-3POS-dueño } & 2 \mathrm{~s}-3 \text { POS-dueño } & \text { 3POS-dueño } \\ \text { 'Soy su dueño' } & \text { 'Eres su dueño' } & \text { 'Es su dueño' }\end{array}$

(89) 'Weenhayek (Claesson 2016: 18)
['o-la-wuuh-uyh $]_{\text {PRED }}$
1s-3Pos-due ño-PL
['inaa-la-wuh-uyh $]_{\mathrm{PRED}}$ 1INCL.S-3POS-dueño-PL
$[\text { 'aa-la-wuh-uyh }]_{\mathrm{PRED}}$
'Somos (excl.) sus dueños'
'Somos (incl.) sus dueños'
2s-3Pos-dueño-PL
'Ustedes son sus dueños'

(90) 'Weenhayek (Claesson 2016: 18) '1 $^{31}$

'o-lhaa-la-wuk

lhaa-la-wh-uy

1S-RR-3POS?-dueño

RR-3POs?-dueño-PL

'Soy dueño de mí mismo/ soy libre'

'Ellos son sus propios dueños/ son libres'

Claesson (2008 y 2017) señala ejemplos de una tercera posibilidad, que utiliza el verbo 'venir'. El autor documenta dos configuraciones. La primera puede considerarse como básica (91a) (venimos-de $+F N$ donde la FN). Además, es posible emplear una construcción con incorporación nominal, puesto que el Wichí/'Wenhaayek es la única lengua mataguaya en la que tal estructura es productiva (91b). Obsérvese que la expresión 'río abajo' está derivada del aplicativo

-(hi)kyà -(h)kyà 'abajo'. La función de la /m/ final es poco clara, pero aparece igualmente en algunos aplicativos de las otras lenguas de la familia mataguaya: nivaĉle, -fitfam 'abajo' y 'río abajo' ( $\neq-$ tyifam 'arriba' y 'río arriba') y -fảm $\sim-\int a m \sim-x a ̉ m \sim-x a m$ 'perlativo; dentro

${ }^{31}$ Claesson aclara que el morfema reflexivo lhaa- aparece con las primera y tercera personas. En el último caso, se podría pensar en una inversión (reflexivo + sujeto en lugar de persona + reflexivo), pero no en el primero, donde se sigue la pauta sujeto + reflexivo, pero no se explica la presencia del elemento la- (v. nota 32). 
del agua o fuego', maká (Gerzenstein 1994: 118-120, 126), y chorote (Carol 2014: 246248, 274-275).

(91a) 'Weenhayek (Claesson 2016: 37)

'o-tàlh-ee-t'at wikyi' tà hikyààm-lhele-yh

1s-venir-ABL-CONF gente SUB río.abajo-habitante-PL

'Somos descendientes de la gente de río abajo (abajeños)'

(91b) 'Weenhayek (Claesson 2016: 37)

'o-lhaam-elh 'o-tàlh-p'an-te-[hikyààm-lhele-y]-eh

1-PRON-PL 1s-venir-EV.NO.VISTO-PREM-[río.abajo-habitante-PL]-ABL

'Nosotros somos descendientes de los antiguos abajeños'

La construcción con 'venir' es posible también por lo menos en variedades wichí occidentales (91c-d).

(91c) Wichí, (SBA 2002, Mateo 22: 42)

$\begin{array}{lllllll}\text { ¿Atsi tä } & \text { lhenayek tälhe? } & \text { - Lhamel yok: } & \text { tälhe } & \text { David } \\ \text { atsi tä } & \text { lhenayek } & \varnothing \text {-tat-e? } & \text { - lh-am-el yok: } & \varnothing \text {-tät-e } & \text { David } \\ \text { cuyo } & \text { suB ENF } & \text { 3s-venir-ABL } & \text { 3-PRON-PL } & \text { 3s.decir } & \text { 3s-venir-ABL } & \text { David } \\ \text { ‘(Díme) pues, de quién es hijo? Ellos dijeron: de David' } & & \end{array}$

(91d) Wichí, (SBA 2002, Apocalipsis)

\begin{tabular}{|c|c|c|c|c|}
\hline wet & tälhe & Aser & laka wichi & doce mil \\
\hline wet & Ø-talh-e & Aser & la-ka-wichi & doce mil \\
\hline & 3s-venir-ABL & Aser & 3POS-CL.POS-gente & doce mil \\
\hline
\end{tabular}

Para las variedades wichí habladas hacia el sur del territorio 'weenhayek, Lunt (1999) documenta tres construcciones, que parcialmente coinciden con las del 'weenhayek. La primera combina una cópula con sujeto de tercera persona con un nombre con poseedor de primera o segunda persona (92a). De acuerdo con este autor, esta construcción sería la única posible para dichas configuraciones. Sin embargo, Terraza (2009), que describe variedades habladas parcialmente en la misma zona que Lunt, documenta también predicados de tercera persona con poseedores de primera y segunda sufijados (92b).

(92a) Wichí (Lunt 1999: 109)

$\begin{array}{ll}\text { häpe } \quad \text {-wuk } & \text { häpe a-wuk } \\ \text { 3s.COP 1POS-dueño } & \text { 3s.COP 2POS-dueño' } \\ \text { 'Es mi dueño' } & \text { 'Es tu dueño' }\end{array}$

(92b) Wichí (Terraza 2009: 64)
la-xuky $a-n u$
la-kila-am
3s-padre-1Pos 3s-hermano.mayor-2Pos
'Es mi padre' 'Es tu hermano mayor' 
La segunda posibilidad es idéntica con la de (84) del 'Weenhayek (93-94).

(93) Wichí (Lunt 1999: 109)

$\begin{array}{lll}o \text {-häpe } a \text {-wuk } & o \text {-häpe le-wuk } & a \text {-häpe } o \text {-wuk } \\ \text { 1s-COP 2POS-dueño } & \text { 1s-COP 3POS-dueño } & \text { 2s-COP 1POS-dueño } \\ \text { 'Soy tu dueño' } & \text { 'Soy su dueño' } & \text { 'Eres mi dueño' }\end{array}$

(94) Wichí (Lunt 1999: 109)

a-hape le-wuk

2s-COP 3POS-dueño

'Eres su dueño'

Para la tercera configuración, hay dos opciones. Se puede prefijar el índice posesivo y sufijar el índice subjeto del predicado (95), o prefijar ambos (96). El estudio de Nercesian (2019) sobre la gran variación interdialectal en la posición del objeto pronominal en wichí/ weenhayek hace suponer que los fenómenos deben estar ligados. En la configuración de segunda persona sujeto y poseedor de primera persona, aparece una le- adventicia entre los dos índices y la base del verbo. Lunt (1999: 109-110) no comenta este fenómeno, y su función no resulta clara (97). En otros ejemplos, le- figura como prefijo posesivo de tercera persona. ${ }^{32}$ Este elemento corresponde al índice de tercera persona la- del 'weenhayek, pero en las combinaciones de segunda persona sujeto con primera posesiva, el 'weenhayek solo emplea la construcción analítica: 'weenhayek 'aa-hâpe 'oo-wuk = wichí $a$-häpe o-wuk opción sintética $o$-a-le-wuk 'eres mi dueño'.

(95) Wichí (Lunt 1999: 109)

a-chen-ek-n'o
2Pos-mandar-NMLZ-1s
'Soy tu mensajero'

le-chen-ek-n'o

3Pos-mandar-NMLZ-1s

'Soy su mensajero'

(96) Wichí (Lunt 1999: 109)

o-le-chen-ek

a-le-chen-ek

1s-3POs-mandar-NMLZ

2s-3POs-mandar-NMLZ

'Soy su mensajero'

'Eres su mensajero'

${ }^{32}$ En una nota al pie, Alvarsson \& Claesson (2014: 450) adviertan un caso similar en verbos transitivos del 'weenhayek. Al prefijarse un sujeto de primera persona y un objeto de segunda, se cuela un índice ' $i$ - de tercera persona entre éstos y la base verbal: 'o- 'aa- 'i-lành [1S-2O-3S-matar] 'yo te mato'. En el paradigma reflexivo, el mismo índice se usa en las primera y tercera personas: 'o-lhaa- ' $i$-lej [1S-RR-3S-lavar] 'me lavo', Ø-lhaa-' $i$-lej [3S-RR-3S-lavar] 'se lava', pero falta, por ejemplo, en otros contextos 'aa-la-lej [RR.2-2S-lavar] 'te lavas'. De acuerdo con Claesson (2017: 17), la combinación de primera persona inclusiva + reflexivo es lhani-. Un prefijo verbal ' $i$ - (no reflexivo) - en vez de 'iyaa- - es también marca de primera persona inclusiva $(1+2)$ de los verbos de la segunda conjugación. Podría también tratarse de una simple alternancia entre base verbal que empieza con consonante y otra que prefija una /i/ epentética. Cualquiera sea su origen, queda por elucidar la contextualización de esta /'i/. Nercesian (2019) nota algunos ejemplos de este fenómeno, segmentándolos de tal manera que la /i/ forma parte del lexema verbal (-ilon 'matar', -ikyen 'mandar', etc.). A estas alturas, esta solución me parece más sensata. 
(97) Wichí (Lunt 1999: 109-110)

$\begin{array}{lll}\text { o-a-le-chen-ek } & o-a-l e-w u k & o-a-l e-h u m n-e k \\ \text { 1POS-2s-3Pos?-mandar-NMLZ } & \text { 1POS-2s-3Pos?-dueño } & \text { 1POS-2s-3Pos?-gustar-NMLZ } \\ \text { 'Eres mi mensajero' } & \text { 'Eres mi dueño' } & \text { 'Eres mi amigo' }\end{array}$

Cuando el índice personal va sufijado a un predicado nominal, representa el sujeto de la construcción, pero cuando el mismo índice aparece ligado a un verbo, desempeña un papel de no sujeto (objeto o cualquier otra función si va seguido por un aplicativo).

\section{Conclusiones}

Se documentaron ocho construcciones de predicación nominal posesiva en nivaĉle y maká. El tipo más simple es el ecuativo, que combina un predicado con una frase nominal posesiva (21) y un pronombre o presentativo con una frase nominal posesiva (22). Una tercera opción combina dos predicados, uno que consiste en un pronombre, y otro en un nombre precedido por un índice posesivo, sin determinante. Recuérdese que solo la presencia de un determinante otorga al nombre su status referencial y formar con éste una frase nominal. De las restantes cinco construcciones, tres están formadas con un nombre, y dos por un verbo. El índice personal del prefijo de los tipos § 3.2.a (nivaĉle) y §3.2. b (maká) es típico de verbos transitivos con $1^{\mathrm{a}}$ persona sujeto $+2^{\mathrm{a}}$ persona objeto (yo te), aunque el alineamiento jerárquico solo mantiene la primera. Sin embargo, en este contexto, el conjunto debe leerse como poseedor de primera persona con sujeto de segunda ( $m i$ [hijo eres] tú). El maká, sin embargo, requiere la presencia del prefijo nominal de poseedor de primera persona, mientras el nivaĉle puede opcionalmente reduplicar la segunda persona como sufijo instrumental. El cuarto tipo forma un predicado de tipo $[\mathrm{POS}-\mathrm{N}]_{\mathrm{PRED}}(\S 3.1)$, en el cual la presencia de un conominal es opcional. El quinto tipo (\$3) marca dos índices [POS-N-S-INST], uno prefijado y otro sufijado junto con el aplicativo instrumental. Este último representa el sujeto de la construcción. Finalmente, los tipos documentados en $\S 3.5$ y $\$ 3.6$ son puros verbos. El verbo nivaĉle -tswt’ax $\sim$ maká $-(a) t s a t^{2} a \chi(\S 3.5)$ es el verbo posesivo 'ser dueño de'. En esta estructura, el prefijo verbal va coindexado con el poseedor, y el verbo va seguido por el índice personal sujeto junto con el aplicativo instrumental. El verbo nivaĉle -ta't $\sim$ maká -tat 'venir' tiene, en la construcción descrita en $\$ 3.6$ el sentido muy restringido de 'ser descendiente de'. De las siete construcciones examinadas del nivaĉle y del maká, tres son tipológicamente muy raras por combinar sintéticamente dos índices personales en una misma palabra para representar una predicación nominal posesiva con nombres de relaciones interpersonales. Como se habrá observado, construcciones similares, con variaciones idiosincráticas, han sido documentadas en todas las lenguas de la familia mataguayo, y una misma configuración puede corresponder a más de una construcción (Tabla 5). 
Abreviaturas

$\mathrm{A}=$ agente; $\mathrm{ABL}=$ ablativo; $\mathrm{AN} . \mathrm{DOM}=$ animal doméstico; $\mathrm{ANAF}=$ anafórico; $\mathrm{BEN}$ $=$ benefactivo/malefactivo; CAUS = causativo; CL.POS = clasificador posesivo; $\mathrm{CMP}=$ compañero; $\mathrm{CON}=$ conativo; $\mathrm{CONF}=$ confirmativo; $\mathrm{CONJ}=$ conjetural; $\mathrm{COP}=$ cópula; CUANT = cuantificador; $\mathrm{D}=$ determinativo ${ }^{33} \mathrm{DEM}=$ demostrativo; DESP $=$ desposesivo; $\mathrm{DIAD}=$ marcador diádico; DIST = distal; EN 'en' (inesivo); $\mathrm{ENF}=$ enfático; $\mathrm{EV}=$ evidencial; $\mathrm{EXCL}=$ exclusivo; $\mathrm{F}$ = femenino; FAC = factitivo; FD = frase determinativa; $\mathrm{FN}=$ frase nominal; HUM = humano; INCL = inclusivo; IINDEF = indefinido; INDIC = indicativo; INST = instrumental; INT = intensivo; IRR = irrealis (realis no marcado); LOC = locativo; $\mathrm{LOC} / \mathrm{DAT}=$ locativo/dativo; $\mathrm{M}=$ masculino; $\mathrm{MED} . \mathrm{POS}=$ mediador posesivo; $\mathrm{N}=$ nombre; $\mathrm{NEG}=$ negativo; $\mathrm{NMLZ}=$ nominalizador; $\mathrm{NO} . \mathrm{HUM}=$ no humano; $\mathrm{O} \sim \mathrm{OBJ}$ = objeto; $\mathrm{P}=$ paciente; $\mathrm{PERL}=$ perlativo; PERS = índice personal sufijado; $\mathrm{PL}=$ plural; PL.P = plural + aplicativo; POS = índice de poseedor; POS.IND = poseedor indefinido; PRED = predicativo; $\mathrm{PREG}=$ pregunta; $\mathrm{PREM}=$ pasado remoto; $\mathrm{PROF}=$ profesional; $\mathrm{PRON}=$ pronombre; $\mathrm{PROSP}=$ prospectivo; $\mathrm{PROX}=$ proximal; $\mathrm{PRT}=$ participio; $\mathrm{RR}$ $=$ reflexivo-recíproco; $\mathrm{REF}=$ referencial; $\mathrm{REL}=$ relator (nombre relacional); $\mathrm{S} \sim \mathrm{SUJ}=$ sujeto; $\mathrm{SEP}=$ separativo; $\mathrm{SG}=$ singular (número de persona prefijado); $\mathrm{SG} . \mathrm{P}=$ singular + aplicativo; $\mathrm{S} . \mathrm{IND}=$ sujeto indefinido; $\mathrm{SUB}_{1}=$ subordinante realis; $\mathrm{SUB}_{2}=$ subordinante irrealis; TOP = topicalizador; $\mathrm{V}=$ verbo; $\mathrm{VBLZ}=$ verbalizador; VENT.ANT = ventivo anticipado.

\section{Referencias}

Alvarsson, Jan-Åke; Kenneth Claesson (2014). 'Weenhayek (mataco). In Mily Crevels; Pieter Muysken (eds.), Lenguas de Bolivia. Tomo III, Oriente, pp. 415-465. La Paz: Plural Editores.

Amith, Jonathan D.; Thomas C. Smith-Stark (1994a). Transitive nouns and split possessive paradigms in central Guerrero Nahuatl. International Journal of American Linguistics 60(4): 342-368.

Amith, Jonathan D.; Thomas C. Smith-Stark (1994b). Predicate nominal and transitive verbal expressions of interpersonal relations. Linguistics 32(3): 511-547.

Brandão, Ana Paula (2018). Nonverbal predication in Paresi-Haliti. In Simon E. Overall; Rosa Vallejos; Spike Gildea (eds.), Nonverbal predication in Amazonian languages, pp. 85-102. Amsterdam: John Benjamins.

Braunstein, José A. (1983). Algunos rasgos de la organización social de los indígenas del Gran Chaco. Trabajos de Etnología, Publicación No. 2. Buenos Aires: Instituto de Ciencias Antropológicas, Facultad de Filosofía y Letras, Universidad de Buenos Aires.

Braunstein, José A. (1993). Territorio e historia de los narradores Matacos. Hacia una Nueva Carta Étnica del Gran Chaco 5: 4-74. Las Lomitas: Centro del Hombre Antiguo Chaqueño (CHACO).

${ }^{33}$ El número que aparece como subíndice indica diferentes series de determinantes que combinan, de acuerdo con cada lengua, rasgos como singular/plural, masculino/femenino, humano/no-humano, distancia y evidenciadidad. Para más detalles, v. Carol (2014: 390-398 [chorote]), Fabre (2016: 87-100 [Nivaĉle]), Gerzenstein (1994: 165-174 [maká]), Gutiérrez 2015 [nivaĉle]). 


\section{FABRE - LA PREDICACIÓN NOMINAL POSESIVA...}

Campbell, Lyle; Luis Díaz; Fernando Ángel (2020). Nivaclé grammar. Salt Lake City: The University of Utah Press.

Carol, Javier (2014). Lengua chorote (mataguayo). Estudio fonológico de morfosintáctico (Lincom Studies in Native American Linguistics 72). Munich: Lincom.

Carpio, María Belén (2012a). Fonología y morfosintaxis de la lengua hablada por grupos tobas en el oeste de Formosa (Argentina) (Lincom Studies in Native American Linguistics 67). Munich: Lincom.

Carpio, María Belén (2012b). Interacción de posesión y número nominal en el toba ñachilamole’k (flia. guaycurú, Formosa, Argentina). Lingüistica 28: 99-118.

Chafe, Wallace L. (2015). A grammar of the Seneca language. University of California Publications, Linguistics, Volume 150.

Claesson, Kenneth 1981-1996. Silààt tà 'is tà 'no'weenho wikyi'(Nuevo Testamento, versión revisada). www.noctenes.org/onewebmedia/NT\%20rev\%20publ.pdg

Claesson, Kenneth (2008). Notas sobre el vocabulario 'Weenhayek. Sociedad Bíblica Boliviana. www.noctenes.org

Claesson, Kenneth (2016). Estudio de la gramática del idioma 'Weenhayek. www.noctenes.org

Claesson, Kenneth (2017). Estudio de la gramática del idioma 'Weenhayek. Segunda parte. Verbos. Marcación de complementos. www.noctenes.org

Danielsen, Swintha (2007). Baure. An Arawak language of Bolivia. Indigenous Languages of Latin America (ILLA) 6. Leiden: CNws Publications.

Danielsen, Swintha (2012). Baure. In Mily Crevels; Pieter Muysken (eds.), Lenguas de Bolivia. Tomo II. Amazonía, pp. 295-339. La Paz: Plural Editores.

Evans, Nicholas (2000). Kinship verbs. In Petra M. Vogel; Bernard Comrie (eds.), Approaches to the typology of word classes, pp.103-172. Berlin: Mouton de Gruyter.

Evans, Nicholas; Lucía Golluscio; Fresia Mellico (2010). La construcción diádica en mapudungun. LIAMES. Linguas Indigenas Americanas 10: 49-66. https://doi.org/10.20396/liames.v10i1.1508

Fabre, Alain (2012). Intersección de alineamiento inverso (jerárquico) y orientación verbal hacia P/T o R en los verbos transitivos del nivaĉle (Chaco paraguayo). LIAMES. Linguas Indígenas Americanas 12: 7-21. https://doi.org/10.20396/liames.v0i12.1484

Fabre, Alain (2016). Gramática de la lengua nivaĉle (familia mataguayo, Chaco paraguayo) (Lincom Studies in Native American Linguistics 78). Munich: Lincom.

Fabre, Alain (2018). Some peculiarities of comparative constructions in Nivaĉle (Mataguayan family, Paraguayan Chaco). Linguistic Discovery 16(1): 14-30. doi:10.1349/PS1.1537-0852.A.482

Gerzenstein, Ana (1994). Lengua Maká. Estudio descriptivo. Buenos Aires: Instituto de Lingüística, Facultad de Filosofía y Letras, Universidad de Buenos Aires. 
Gerzenstein, Ana (1999). Diccionario etnolingüístico Maká-Español (DELME). Buenos Aires: Instituto de Lingüística, Facultad de Filosofía y Letras, Universidad de Buenos Aires.

Gerzenstein, Ana; Beatriz Gualdieri (2003). La armonía vocálica en lenguas chaqueñas de las familias Guaycurú y Mataguaya. LIAMES. Linguas Indigenas Americanas 3: 99-112. https://doi.org/10.20396/liames.v3i1.1415

González, Raúl Eduardo (2013). Reorganización del sistema de número en toba (familia guaycurú). UniverSOS 10: 127-139.

González, Raúl Eduardo (2016). Codificación de las categorías de "grupo" y "grupo restringido" en toba del este de Formosa, Argentina. LIAMES. Linguas Indigenas Americanas 16(2): 221-240. https://doi.org/10.20396/liames.v16i2.8645931

Gutiérrez, Analía (2015). Evidential determiners in Nivaĉle. Anthropological Linguistics 57(4): 412-443.

Halpern, A.M. 1942. Yuma kinship terms. American Anthropologist 44: 425-441. https://www.jstor.org/stable/663465

Haspelmath, Martin (2013). Argument indexing: a conceptual framework for the syntactic status of bound person forms. In Dikk Bakker; Martin Haspelmath (eds.), Languages across boundaries. Studies in memory of Anna Siewierska, pp. 197-226. Berlin: De Gruyter.

Hunt, R.J. (1940). Mataco grammar. Tucumán: Instituto de Antropología, Universidad Nacional de Tucumán [redactado antes de 1925, regreso de Hunt a Inglaterra].

Koenig, Jean-Pierre; Karin Michelson (2010). Argument structure of Oneida kinship terms. International Journal of American Linguistics 76(2): 169-205.

Lunt, Roberto M. (1999). Wichi lhämtes. Una gramática del idioma wichí con ejercicios. Tartagal (Salta): ASOCIANA.

McGregor, William B. (1996). Dyadic and polyadic kin terms in Gooniyandi. Anthropological Linguistics 38(2): 216-247.

Messineo, Cristina; Ana Gerzenstein (2007). La posesión en dos lenguas indígenas del Gran Chaco: toba (guaycurú) and maká (mataguayo). LIAMES. Línguas Indígenas Americanas 7: 61-79. https://doi.org/10.20396/liames.v7i1.1455

Miller, Amy (1991). The Jamul Diegueño predicate nominal construction. Linguistic notes from La Jolla 16: $71-88$.

Miller, Amy (2001). A grammar of Jamul Tiipay. Berlin: Mouton de Gruyter.

Mithun, Marianne (1999). The languages of Native North America. Cambridge: Cambridge University Press.

Mithun, Marianne (2017). Argument marking in the polysynthetic verb and its implications. In Michael Fortescue; Marianne Mithun; Nicholas Evands (eds.), The Oxford handbook of polysynthesis, pp. 30-58. Oxford: Oxford University

Moser, Mary B.; Stephen A. Marlett (1989). Terminología de parentesco seri. Anales de Antropología 26: 367388. 


\section{FABRE - LA PREDICACIÓN NOMINAL POSESIVA...}

Nercesian, Verónica (2014). Wichi lhomtes. Estudio de la gramática y la interacción fonología-morfologíasintaxis-semántica (Lincom Studies in Native American Linguistics 74). Munich: Lincom.

Nercesian, Verónica (2019). Variación dialectal y diacrónica del objeto pronominal en wichí/ weenhayek (mataguayo): paradigma prefijante y sufijante. Cuadernos de lingüística de El Colegio de México 6(1): $1-73$.

Nichols, Johanna (2017). Polysynthesis and head marking. In Michael Fortescue; Marianne Mithun; Nicholas Evans (eds.), The Oxford handbook of polysynthesis, pp. 59-69. Oxford: Oxford University Press.

Queixalós, Fransisco (1998). Nom, verbe et prédicat en sikuani. Paris: Peeters.

Queixalós, Francisco (2000). Syntaxe sikuani. Paris: Peeters.

Rose, Françoise (2018). Nonverbal predication and the nonverbal clause type of Mojeño Trinitario. In Simon E. Overall; Rosa Vallejos; Spike Gildea (eds.), Nonverbal predication in Amazonian languages, pp. 53-83. Amsterdam: John Benjamins.

Sapir, Edward (1917). Uhlenbeck, C.C. 'Het identificeerend karakter der possessieve flexie in talen van NoordAmerika' ('The identifying character of the possessive inflection in languages of North America'). Reprinted from 'Verslagen en Mededeelingen der Koninklijke Akademie van Wetenschappen, Afdeeling Letterkunde, $5^{\mathrm{e}}$ Reeks, Deel II: 345-371. Amsterdam, 1918. Inter-national Journal of American Linguistics 1(1): $86-90$.

SBA = Sociedad Bíblica Argentina (1997). Sinia'Jlamtis. El Nuevo Testamento en el idioma Iyojwa 'ja (Chorote). Buenos Aires.

SBA = Sociedad Bíblica Argentina (2002). Dios Lhämtes tä Matchehen. Buenos Aires.

SBP = Sociedad Bíblica del Paraguay (1994). Pa Dios tasinôyjoom nôqueesh. Nava Dios lhasinôc na nivacle lhcliish. Asunción.

Seelwische, José (1975). Na lhasinônash napi nivacle. Gramática nivacle. Asunción: El Gráfico.

Seelwische, José (1990). Diccionario nivacle. Nivacle-castellano. Castellano-nivacle. Mariscal Estigarribia: Vicariato Apostólico del Pilcomayo.

Seelwische, José (1995). Sui papi catsinôvot p'alhaa ti yisclansha 'ne na lhcootsjat. Lecturas de historia para el $5^{\circ}$ grado. Mariscal Estigarribia: Vicariato Apostólico del Pilcomayo.

Seiler, Hansjakob (1982). Inherent vs. established relation, proximity versus obviation, and two types of Cahuilla kinship expressions. International Journal of American Linguistics 48(2): 185-196.

Seiler, Hansjakob (1983). Possession as an operational dimension of language. Tübingen: Gunter Narr Verlag.

Smeets, Ineke (2007). A grammar of Mapuche. Berlin: Mouton de Gruyter.

Stassen, Leon (2009). Predicative possession. Oxford: Oxford University Press.

Stell, Nélida Noemi (1987). Gramática descriptiva de la lengua Niwakle (Chulupí), Vol. 1-2 (Tesis doctoral). Facultad de Filosofía y Letras, Universidad Nacional de Buenos Aires.

http://repositorio.filo.uba.ar/handle/filodigital/1898 
Terraza, Jimena (2009). Grammaire du wichi: phonologie et morphosyntaxe (Thèse de Doctorat). Université du Québec à Montréal.

Tierra Libre (2014). Pa lhtistenjayish lhapesh napi nivaĉle. Asunción.

Troike, Rudolf C. (1996). Sketch of Coahuilteco, a language isolate of Texas. In Ives Goddard (ed.), Handbook of North American Indians, Volume 17. Languages, pp. 644-665. Washington: Smithsonian Institution.

Vázquez Soto, Verónica (2013). Predicación de términos de parentesco en cora meseño. In Enrique Palancar; Roberto Zavala Maldonado (eds.), Clases léxicas, posesión y cláusulas complejas en lenguas d $e$ Mesoamérica, pp. 77-102. México, D.F.: Publicación de la Casa Chata, CIESA.

Vidal, Alejandra (2001). Pilagá grammar (Guaykuruan family, Argentina (Ph.D. dissertation), University of Oregon.

WBT = Wycliffe Bible Translators (2013). Intata Łe'lijei. El Nuevo Testamento en Maká de Paraguay.

Wicke, Charles R.; Miguel Chase-Sardi (1969a). Componential analyses of Chulupí (Ashluslay) kinship terminology. Ethnology 8(4): 484-493.

Wicke, Charles R.; Miguel Chase-Sardi (1969b). Análisis componencial de la terminología de parentesco chulupí (ashluslay). Revista del Ateneo Paraguayo 4(2): 185-202.

Wolfart, H.C. (1996). Sketch of Cree, an Algonquian language. In Ives Goddard (ed.), Handbook of North American Indians, Volume 17. Languages: 390-439. Washington: Smithsonian Institution.

Zavala Maldonado, Roberto (2016). Construcciones posesivas biactanciales en oluteco. Zarina Estrada Fernández (ed.), Memorias del VIII Encuentro de Lingüistica en el Noroeste, tomo 1: 353-377. Hermosillo: unIson.

Recebido: $18 / 8 / 2020$

Versão revista e aceita: 10/10/2020

Publicado: 27/10/2020

LIAMES, Campinas, SP, v. 20, 1-45, e020014, 2020 